\title{
Simple Relationships for the Estimation of Melting Temperatures of Homologous Series
}

\author{
J ames S. Chickos* and Gary Nichols
}

Department of Chemistry, University of Missouri-St. Louis, St. Louis, Missouri 63121

\begin{abstract}
The melting behavior of a homologous series is described in terms of the melting of the parent molecule and of the polymer the series eventually forms. For those series characterized by a parent melting below the melting temperature of the related polymer, the melting behavior can be described quantitatively by the hyperbolic function $T_{f}(n)=T_{f}(\infty)[1-1 /(m n+b)]$, where $T_{f}(n)$ refers to the melting temperature of a compound with $n$ repeat units, $T_{f}(\infty)$ is the melting temperature of the polymer, and $m$ and $b$ are two variables used in fitting the data. A plot of $\left[1 /\left(1-T_{f}(n) / T_{f}(\infty)\right)\right]$ against $n$ results in a straight line with slope $m$ and intercept $b$. This linear relationship provided the analytical form of the equation described above. For series with parents exhibiting melting temperatures higher than those of the related polymer, a linear correlation is observed when $\left[1 /\left(1-T_{f}(\infty) / T_{f}(n)\right)\right]$ is plotted against $(n)$. This resulted in the hyperbolic relationship $T_{f}(n)=T_{f}(\infty) /[1-1 /(m n+b)]$. These equations appear applicable for the quantitative evaluation of the melting behavior of any homologous series, provided care is taken to consider compounds characterized by the same symmetry number. Molecules containing odd and even numbers of repeat groups are generally treated separately. The hyperbolic behavior exhibited by the melting temperature in most series appears characteristic of molecules that seem to pack similarly in the solid state. Series with members exhi biting liquid-crystal behavior are successfully modeled by these equations, provided the transition correlated is the temperature at which theliquid becomes isotropic. The usefulness of these equations was tested by selecting three data points from each series to provide values for $\mathrm{m}$ and b. The melting temperatures of most compounds in the series were estimated using these parameters. This resulted in a standard deviation of $\pm 6.6 \mathrm{~K}$ between experimental and calculated values based on a total of 995 compounds.
\end{abstract}

\section{Introduction}

The melting temperature of a crystalline substance is a fundamental physical property of the material. The study of the behavior of melting as a function of structure has been of perennial interest. ${ }^{1} \mathrm{~A}$ number of studies have demonstrated that the melting temperature of linear molecules is not an additive property but one that does vary in a continuous fashion with structure. Theories of the dependence of melting on molecular weight for linear molecules, particularly as applied to polymers, have been developed by Flory2,3 and others. ${ }^{4,5}$ Several different relationships have been derived to correlate the melting behavior of the even n-alkanes shown in Figure 1.6,7

The development of a general protocol for the estimation of melting temperatures has proven to be elusive. Recent efforts by Yalkowsky et al. ${ }^{8-10}$ using several different approaches that have induded relationships between boiling and melting temperatures and modifications of Walden's Rule have yielded estimates with standard errors of approximately $\pm 30 \mathrm{~K}$. Katritzky and co-workers ${ }^{11}$ have published quantitative structure-property relationship studies in which the melting temperatures of 142 substituted pyridines were correlated with six molecular descriptors with a correlation of $r^{2}=0.8568$. Efforts to find correlations between melting temperature and properties such as crystal density or packing energy have not proven very successful. ${ }^{12}$ This work describes the melting behavior observed for a variety of different compounds examined as a collection of molecules in a homologous series. It proposes some simple equations that can be used to successfully

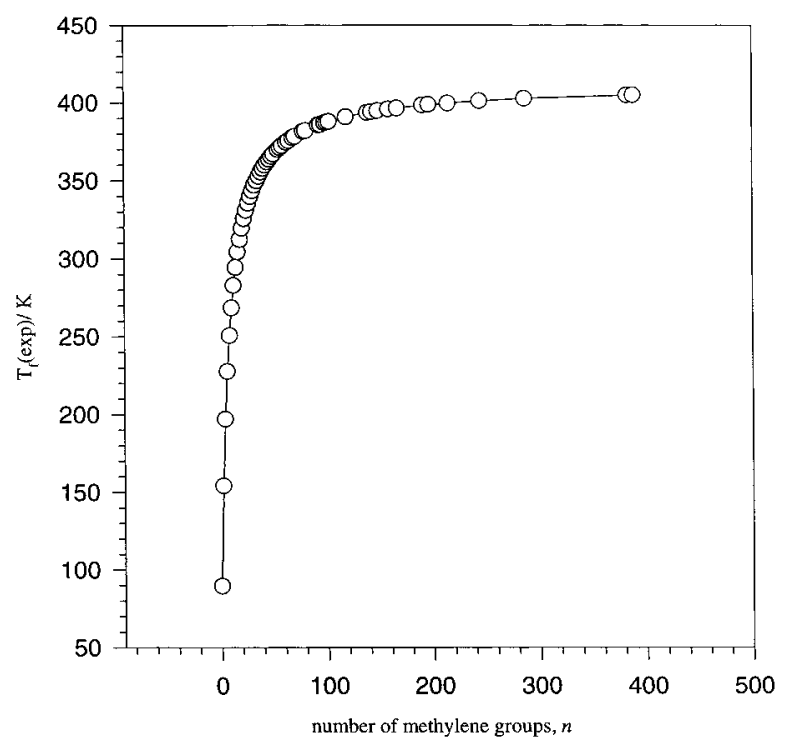

Figure 1. Melting temperatures of the even $n$-alkanes versus the number of methylene groups: (circles) experimental data; (line) calculated results.

reproduce the melting behavior of each series. In addition, a simple protocol is proposed that will provide reasonably good estimates of the melting behavior of the remaining members of the series when data for only a few members of the series are available. The method appears generally applicable to series differing widely in structure and in melting behavior. 


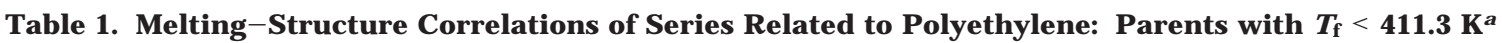

\begin{tabular}{|c|c|c|c|c|c|c|c|c|}
\hline homologous series & parent compound & $\begin{array}{c}\mathrm{T}_{\mathrm{f}} / \mathrm{K} \\
\text { parent }\end{array}$ & S & $\mathrm{m}$ & $\mathrm{b}$ & $r^{2}$ & $\pm \sigma / \mathrm{K}$ & $\mathrm{n}_{\mathrm{T}}$ \\
\hline \multicolumn{9}{|c|}{ A. Hydrocarbons } \\
\hline \multirow[t]{2}{*}{ n-alkanes ${ }^{b}$} & butane & 134.9 & e & 0.161 & 1.153 & 0.989 & 2.0 & 53 \\
\hline & propane & 85.2 & 0 & 0.172 & 0.948 & 0.994 & 3.5 & 24 \\
\hline \multirow[t]{2}{*}{ 1-alkenesc } & 1-pentene & 107.9 & $\mathrm{e}$ & 0.170 & 0.856 & 0.999 & 7.2 & 9 \\
\hline & 1-butene & 87.8 & 0 & 0.164 & 0.925 & 0.998 & 2.4 & 8 \\
\hline \multirow{2}{*}{ 2-methylalkanesc } & 2-methylpentane & 119.6 & $\mathrm{e}$ & 0.155 & 0.951 & 0.993 & 5.5 & 10 \\
\hline & 2-methylbutane & 113.4 & 0 & 0.144 & 1.18 & 0.998 & 2.1 & 9 \\
\hline \multirow[t]{2}{*}{ 3-methylalkanesc } & 3-methylhexane & 100.2 & $\mathrm{e}$ & 0.145 & 0.981 & 0.984 & 4.8 & 6 \\
\hline & 3-methylheptane & 152.7 & 0 & 0.129 & 1.19 & 0.996 & 2.4 & 7 \\
\hline \multirow{2}{*}{ 4-methylalkanes ${ }^{c}$} & 4-methylheptane & 152.2 & $\mathrm{e}$ & 0.125 & 1.29 & 0.998 & 1.5 & 6 \\
\hline & 4-methyldecane ${ }^{d}$ & 195.7 & 0 & 0.128 & 1.23 & 0.995 & 2.1 & 7 \\
\hline \multirow{2}{*}{ 5-methylalkanese } & 5-methyldecane ${ }^{d}$ & 183.2 & $\mathrm{e}$ & 0.121 & 1.24 & 0.995 & 1.9 & 7 \\
\hline & 5-methylnonane & 186.7 & 0 & 0.113 & 1.41 & 0.996 & 2.0 & 6 \\
\hline 2,3-dimethylalkanesc & 2,3-dimethyldecane ${ }^{d}$ & 183.7 & $\mathrm{e}$ & 0.155 & 0.898 & 0.989 & 4.0 & 5 \\
\hline & 2,3-dimethylheptane & 156 & 0 & 0.15 & 0.884 & 0.991 & 7.4 & 6 \\
\hline 2,4-dimethylalkanesc & 2,4-dimethylundecane d $^{\mathrm{d}}$ & 197.7 & $\mathrm{e}$ & 0.136 & 1.13 & 0.992 & 2.6 & 5 \\
\hline & 2,4-dimethyldecane ${ }^{d}$ & 183.2 & 0 & 0.128 & 1.16 & 0.997 & 2.0 & 6 \\
\hline 2,4,6-trimethylalkanese & 2,4,6-trimethyltridecane ${ }^{d}$ & 171.2 & e & 0.151 & 0.781 & 0.962 & 7.5 & 4 \\
\hline & 2,4,6-trimethyldodecane $\mathrm{d}^{\mathrm{d}}$ & 161.2 & 0 & 0.114 & 1.04 & 0.957 & 7.8 & 4 \\
\hline n-alkylcyclopentanes ${ }^{f}$ & propylcyclopentane & 155.8 & e & 0.155 & 1.23 & 0.999 & 0.6 & 8 \\
\hline & ethylcyclopentane & 134.7 & 0 & 0.155 & 1.17 & 0.999 & 1.6 & 8 \\
\hline n-alkylcyclohexanes ${ }^{f}$ & propylcyclohexane & 178.3 & e & 0.165 & 1.45 & 0.999 & 0.6 & 7 \\
\hline & ethylcyclohexane & 161.4 & 0 & 0.164 & 1.47 & 0.999 & 4.1 & 9 \\
\hline n-alkylbenzenes ${ }^{c}$ & propylbenzene & 173.6 & $\mathrm{e}$ & 0.166 & 1.21 & 0.999 & 1.4 & 8 \\
\hline & ethylbenzene & 178 & 0 & 0.164 & 1.23 & 0.999 & 3.4 & 10 \\
\hline 1-alkylnaphthalenesg & 1-propylnaphthalene & 263.2 & $\mathrm{e}$ & 0.190 & 1.67 & 0.997 & 1.4 & 4 \\
\hline & 1-ethylnaphthalene & 259.3 & 0 & 0.171 & 1.77 & 0.998 & 6.7 & 6 \\
\hline 2-alkylnaphthalenes 9 & 2-propylnaphthalene & 270.2 & $\mathrm{e}$ & 0.131 & 2.29 & 0.955 & 3.5 & 5 \\
\hline & 2-ethylnaphthalene & 265.7 & 0 & 0.149 & 2.15 & 0.987 & 6.8 & 6 \\
\hline alkynes $^{f}$ & 1-pentyne & 167.5 & e & 0.172 & 1.15 & 0.999 & 0.7 & 9 \\
\hline & 1-butyne & 147.5 & 0 & 0.180 & 0.993 & 0.999 & 2.0 & 9 \\
\hline & B. Cycloall & & & & & & & \\
\hline cycloalkanes ${ }^{h}$ & & & & 0.188 & 1.18 & 0.856 & 21 & 46 \\
\hline & C. Functionaliz & Alkanes & & & & & & \\
\hline 1-alkanolsi & propanol & 147.2 & $\mathrm{e}$ & 0.239 & 0.968 & 0.998 & 1.9 & 18 \\
\hline & ethanol & 143.2 & 0 & 0.244 & 0.953 & 0.999 & 4.0 & 15 \\
\hline 2-alkanolsj & 2-nonanold & 184.7 & e & 0.257 & 0.87 & 0.992 & 3.1 & 6 \\
\hline & 2-butanol & 158.5 & o & 0.244 & 1.22 & 0.999 & 1.1 & 9 \\
\hline 1-alkanethiolsc & 1-ethanethiol & 125.9 & 0 & 0.153 & 1.12 & 0.998 & 2.6 & 8 \\
\hline methyl alkanoatesk & methyl hexanoate & 202.2 & $\mathrm{e}$ & 0.179 & 1.30 & 0.995 & 2.6 & 17 \\
\hline & methyl propanoate & 185.2 & 0 & 0.167 & 1.26 & 0.991 & 4.1 & 11 \\
\hline alkyl ethanoates ${ }^{c}$ & propyl ethanoate & 178.2 & $\mathrm{e}$ & 0.161 & 1.22 & 0.999 & 3.1 & 8 \\
\hline & ethyl ethanoate & 189.6 & 0 & 0.155 & 1.28 & 0.999 & 7.2 & 9 \\
\hline ethyl alkanoates ${ }^{i}$ & ethyl butanoate & 172.4 & e & 0.166 & 1.28 & 0.999 & 1.4 & 18 \\
\hline primary alkylamines ${ }^{c}$ & propylamine & 190.2 & $\mathrm{e}$ & 0.178 & 1.55 & 0.999 & 0.4 & 8 \\
\hline & ethylamine & 192.2 & o & 0.188 & 1.65 & 0.999 & 0.8 & 10 \\
\hline n-alkanalc & butanal & 176.8 & e & 0.159 & 1.77 & 0.982 & 7.4 & 7 \\
\hline & propanal & 193.2 & o & 0.183 & 1.24 & 0.945 & 8.1 & 8 \\
\hline n-alkanoic acids j & butanoic acid & 268.5 & $\mathrm{e}$ & 0.270 & 1.72 & 0.998 & 1.2 & 18 \\
\hline & propanoic acid & 253.5 & o & 0.265 & 1.44 & 0.999 & 1.0 & 15 \\
\hline 1-chloroalkanesc & 1-chloropropane & 150.2 & e & 0.160 & 1.07 & 0.997 & 2.4 & 8 \\
\hline & chloroethane & 137.2 & o & 0.166 & 0.941 & 0.999 & 6.5 & 9 \\
\hline 1-fluoroalkanesc & 1-fluorotridecane $e^{d}$ & 276.2 & $\mathrm{e}$ & 0.183 & 0.839 & 0.999 & 0.3 & 4 \\
\hline & 1-fluoroethane & 130 & o & 0.171 & 0.846 & 0.999 & 7.3 & 9 \\
\hline 1-bromoalkanes ${ }^{f}$ & 1-bromopropane & 163.2 & $\mathrm{e}$ & 0.164 & 1.15 & 0.999 & 0.9 & 9 \\
\hline & bromoethane & 154.6 & 0 & 0.159 & 1.04 & 0.999 & 4.9 & 11 \\
\hline 1-iodoalkanes ${ }^{f}$ & 1-iodopropane & 171.9 & $\mathrm{e}$ & 0.172 & 1.21 & 0.999 & 2.4 & 18 \\
\hline & iodoethane & 162.1 & o & 0.168 & 1.10 & 0.999 & 3.0 & 19 \\
\hline 1-cyanoalkanesc & 1-cyanopropane & 161.3 & $\mathrm{e}$ & 0.203 & 1.03 & 0.999 & 1.1 & 8 \\
\hline & cyanoethane & 180.3 & o & 0.191 & 1.09 & 0.998 & 2.9 & 9 \\
\hline 1,2-dihydroxyalkanesc & 1,2-hexanediol & 318.2 & 0 & 0.336 & 2.18 & 0.995 & 5.2 & 7 \\
\hline 1-N-methylaminoalkanesc & methyl-n-butylamine & 198.2 & o & 0.164 & 1.55 & 0.994 & 2.2 & 8 \\
\hline 1-N,N-dimethylaminoalkanes ${ }^{c}$ & dimethyl-n-ethylamine & 133.2 & 0 & 0.165 & 0.774 & 0.999 & 0.3 & 7 \\
\hline 2-alkanonesc & 2-pentanone & 195.2 & e & 0.220 & 1.51 & 0.999 & 0.7 & 7 \\
\hline & 2-butanone & 186.2 & 0 & 0.220 & 1.51 & 0.999 & 1.9 & 8 \\
\hline alkyl phenyl ketonesk & acetophenone & 293.2 & o & 0.213 & 2.44 & 0.995 & 0.8 & 5 \\
\hline $\mathrm{F}\left[\mathrm{CF}_{2}\right]_{12}\left[\mathrm{CH}_{2}\right]_{\mathrm{n}} \mathrm{H}^{\mathrm{h}}$ & $\mathrm{F}[\mathrm{CF} 2]_{12}\left[\mathrm{CH}_{2}\right]_{2} \mathrm{H}$ & 344.2 & $\mathrm{e}$ & 0.172 & 5.93 & 0.920 & 1.5 & 9 \\
\hline $\mathrm{N}$-methylalkanamides & $\mathrm{N}$-methylbutanamide & 268 & $\mathrm{e}$ & 0.461 & 1.37 & 0.999 & 0.8 & 7 \\
\hline & $\mathrm{N}$-methylpropanamide & 230.2 & o & 0.435 & 1.13 & 0.999 & 0.6 & 7 \\
\hline 2-hydroxyethylalkanamides' & $\mathrm{N}$-(2-hydroxyethyl)hexanamide & 319.2 & $\mathrm{e}$ & 0.435 & 2.93 & 0.967 & 2.1 & 6 \\
\hline & $\mathrm{N}$-(2-hydroxyethyl)pentanamide ${ }^{d}$ & 305.2 & o & 0.639 & 1.71 & 0.993 & 1.4 & 5 \\
\hline p-chlorophenacylalkanoates' & p-chlorophenacylbutanoate & 328.2 & $\mathrm{e}$ & 0.288 & 3.27 & 0.953 & 6.0 & 6 \\
\hline & p-chlorophenacylpropionate & 371.4 & o & 0.231 & 4.05 & 0.809 & 8.8 & 7 \\
\hline $\mathrm{N}$-octadecylalkanamides ${ }^{\mathrm{m}}$ & $\mathrm{N}$-octadecylbutanamide & 349.7 & $\mathrm{e}$ & 0.257 & 5.49 & 0.981 & 1.3 & 7 \\
\hline
\end{tabular}


564 J ournal of Chemical and Enginering Data, Vol. 46, No. 3, 2001

Table 1 (Continued)

\begin{tabular}{|c|c|c|c|c|c|c|c|c|}
\hline homologous series & parent compound & $\begin{array}{c}\mathrm{T}_{\mathrm{f}} / \mathrm{K} \\
\text { parent }\end{array}$ & $\mathrm{S}$ & $\mathrm{m}$ & b & $r^{2}$ & $\pm \sigma / \mathrm{K}$ & $\mathrm{n}_{\mathrm{T}}$ \\
\hline \multicolumn{9}{|c|}{ C. Functionalized Alkanes (Continued) } \\
\hline \multirow[t]{2}{*}{ n-alkanamides ${ }^{n}$} & butanamide & 389.2 & e & 0.226 & 9.93 & 0.706 & 3.5 & 12 \\
\hline & propanamide & 356.2 & 0 & 0.238 & 8.61 & 0.732 & 5.0 & 7 \\
\hline \multirow[t]{2}{*}{ alkyl 4-nitrobenzoates ${ }^{\circ}$} & propyl 4-nitrobenzoate & 308.2 & $\mathrm{e}$ & 0.162 & 2.22 & 0.995 & 3.0 & 7 \\
\hline & ethyl 4-nitrobenzoate & 330.2 & 0 & 0.213 & 1.94 & 0.984 & 6.9 & 9 \\
\hline n-alkyl 3,5-dinitrobenzoates ${ }^{\circ}$ & ethyl 3,5-dinitrobenzoate & 367.2 & 0 & 0.035 & 5.13 & 0.566 & 2.7 & 8 \\
\hline \multirow[t]{2}{*}{ 1, $\omega$-dihydroxyalkanesc } & 1,2-dihydroxyethane & 260.2 & $\mathrm{e}$ & 0.421 & 1.87 & 0.988 & 1.9 & 8 \\
\hline & 1,3-dihydroxypropane & 246.2 & 0 & 0.476 & 0.25 & 0.993 & 8.1 & 6 \\
\hline \multirow[t]{2}{*}{$\mathrm{N}$-( $\beta$-naphthyl)alkanamides ${ }^{\mathrm{m}}$} & $\mathrm{N}$-( $\beta$-naphthyl $)$ hexanamide & 380.2 & $\mathrm{e}$ & 0.400 & 9.07 & 0.970 & 1.2 & 6 \\
\hline & hthyl)pentanamide & 385.2 & 0 & 0.356 & 9.28 & 0.998 & 3.2 & 3 \\
\hline \multirow[t]{2}{*}{$1, \omega$-alkanedioic acids ${ }^{k}$} & 1,5-undecanedioic acid ${ }^{d}$ & 378 & 0 & 0.730 & 9.30 & 0.925 & 1.9 & 8 \\
\hline & \multicolumn{8}{|c|}{ D. Symmetrically Substituted Derivatives ${ }^{q}$} \\
\hline sym-dialkyl ether ${ }^{c, p}$ & diethyl ether & 157.2 & e & 0.135 & 0.932 & 0.999 & 1.7 & 4 \\
\hline \multirow[t]{2}{*}{ sym-n-alkanoic acid anhydrides } & butanoic anhydride & 198.2 & e & 0.319 & 1.05 & 0.999 & 1.4 & 10 \\
\hline & anhydride & 228.2 & 0 & 0.221 & 2.25 & 0.980 & 23.8 & 5 \\
\hline sym-di-n-alkyl sulfides ${ }^{r, p}$ & diethyl sulfide & 171.2 & 0 & 0.292 & 1.01 & 0.998 & 1.4 & 6 \\
\hline \multirow[t]{2}{*}{ sym-N,N-dialkylamines ${ }^{c, p}$} & diethylamine & 181 & e & 0.298 & 1.14 & 0.913 & 10.2 & 8 \\
\hline & & 210.2 & o & 0.320 & 1.08 & 0.999 & 0.9 & 8 \\
\hline sym-tri-n-alkylamines, & triethylamine & 158.5 & 0 & 0.249 & 0.655 & 0.998 & 1.6 & 7 \\
\hline sym-1,2,3-glycerol trialkanoate ${ }^{s, p}$ & glycerol tridecanoate $^{d}$ & 304.8 & e & 0.296 & 1.50 & 0.999 & 0.5 & 7 \\
\hline$\beta$ form & thermodynamic form & 261.7 & & 0.272 & 0.598 & 0.999 & 1.1 & 7 \\
\hline$\alpha$ form & kinetic form & 290.0 & & 0.263 & 1.31 & 0.999 & 0.8 & 7 \\
\hline$\beta^{\prime}$ form & intermediate for & & & & & & & \\
\hline 1,3-glycerol dialkanoate-2-caproates & glycerol 1,3-dilaurate-2-caproate ${ }^{d}$ & 310.6 & $\mathrm{e}$ & 0.170 & 2.3 & 0.992 & 0.8 & 4 \\
\hline 1,3-glycerol dialkanoate-2-dodecanoates & glycerol 1,3-didecanoate-2-dodecanoate ${ }^{d}$ & 310.7 & e & 0.144 & 2.93 & 0.994 & 0.8 & 4 \\
\hline 1,2-glycerol dialkanoate-3-decanoate ${ }^{s}$ & glycerol 1,2-didodecanoate-3-decanoate & 307.3 & e & 0.129 & 2.74 & 0.741 & 4.1 & 4 \\
\hline
\end{tabular}

a Melting temperatures reported are for the racemic compounds; $\mathrm{S}$, even (e) and odd series (o) of $\mathrm{n}$; $\mathrm{r}^{2}$, correlation coefficient; $\pm \sigma$, standard deviation; $\mathrm{n}_{\mathrm{T}}$, total number of data points. ${ }^{\mathrm{b}}$ References 22 and 23 . ${ }^{\mathrm{c}}$ Reference $16 .{ }^{\mathrm{d}}$ The first available member of the series;

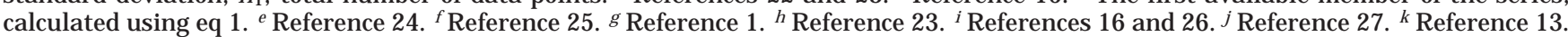
' Reference 28. ${ }^{\mathrm{m}}$ Reference 29. ${ }^{\mathrm{n}}$ References 28,29 , and 32. ${ }^{\circ}$ References 14 and 30. ${ }^{\mathrm{p}}$ Calculated on the basis of the number of methylene groups on a single chain. ${ }^{q}$ References 26 and $29 .{ }^{r}$ References 15,26 , and $31 .{ }^{s}$ Reference 18.

Table 2. Melting-Structure Correlations of Series Related to Polyethylene: Parents with a $T_{f}>411.3 K^{a}$

\begin{tabular}{|c|c|c|c|c|c|c|c|c|}
\hline homologous series & parent compound & $\begin{array}{c}\mathrm{T}_{\mathrm{f}} / \mathrm{K} \\
\text { parent }\end{array}$ & $\mathrm{S}$ & $\mathrm{m}$ & $b$ & $r^{2}$ & $\pm \sigma / \mathrm{K}$ & $\mathrm{n}_{\mathrm{T}}$ \\
\hline \multirow[t]{2}{*}{ 4-alkoxybiphenyl-4-carboxylic acids ${ }^{\mathrm{b}, \mathrm{c}}$} & 4'-ethoxybiphenylcarboxylic acid & 574.7 & 0 & 0.051 & 3.05 & 0.996 & 2.1 & 7 \\
\hline & ronovybinher & 560.2 & $\mathrm{e}$ & 0.062 & 3.02 & 0.997 & 0.5 & \\
\hline alkyl 4'-methoxybiphen & boxyla & 440.7 & 0 & 1.64 & 4.38 & 0.986 & 2.1 & 6 \\
\hline 4-alkyl-3-nitrobiphenyl & 4-ethyl-3-nitrobi phenyl-4'-carboxylic acid & 552 & 0 & 0.074 & 4.03 & 0.997 & 1.3 & 7 \\
\hline 4-alkyl-3-bromobiphenyl-4'-carboxylic acidb,c & 4-ethyl-3-bromobiphenyl-4'-carboxylic acid & 527 & 0 & 0.095 & 3.58 & 0.998 & 0.7 & \\
\hline 8-alkyltheophyllines ${ }^{d}$ & & 534.3 & e & & 0.824 & 0.975 & 4.1 & \\
\hline \multirow[t]{2}{*}{ 4-alkoxybenzoic acids b,e } & cid & 427.2 & $\mathrm{e}$ & 0.530 & 7.38 & 0.990 & 0.5 & 7 \\
\hline & & 469.2 & 0 & 0.632 & 6.09 & 0.991 & 1.0 & 8 \\
\hline$d s^{f}$ & benzoic acid & 416.7 & 0 & 5.25 & -8.4 & 0.964 & 1.5 & \\
\hline \multirow{2}{*}{ dialkylarsinic acids ${ }^{g}$} & & 408 & $\mathrm{e}$ & 0.638 & 16. & 0.813 & 4.4 & \\
\hline & & 411 & 0 & 1. & 11 & 0.987 & 1.1 & \\
\hline $4,4^{\prime}-d$ & 4 & 440.7 & 0 & 1. & 8.96 & 0.956 & 1.0 & 6 \\
\hline \multirow{2}{*}{ 6-alkoxy-2-naphthoic acidsi } & 6 & 497.2 & 0 & 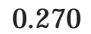 & 3.74 & 0.993 & 2.2 & \\
\hline & & 481.7 & $\mathrm{e}$ & & 4.29 & 0. & 0.7 & \\
\hline & & 461.2 & $\mathrm{e}$ & 1. & 6.62 & 0.921 & 4.3 & \\
\hline $\mathrm{C}_{3} \mathrm{H}_{7} \mathrm{CONH}\left(\mathrm{CH}_{2}\right)_{n} \mathrm{NHCOC}_{3} \mathrm{H}_{7} \mathrm{~K}^{2}$ & $\mathrm{ONH}\left(\mathrm{CH}_{2}\right)_{2} \mathrm{NHCOC}_{3} \mathrm{H}_{7}$ & 465 & $\mathrm{e}$ & 0.826 & 5.73 & 0.959 & 2.7 & \\
\hline $\mathrm{C}_{5} \mathrm{H}_{11} \mathrm{CONH}\left(\mathrm{CH}_{2}\right)_{\mathrm{n}} \mathrm{NHCOC}{ }_{5} \mathrm{H}_{11} \mathrm{k}$ & $\mathrm{C}_{5} \mathrm{H}_{11} \mathrm{CONH}\left(\mathrm{CH}_{2}\right)_{2} \mathrm{NHCOC}_{5} \mathrm{H}_{11}$ & 451 & e & 0.697 & 7.18 & 0.971 & 2.9 & \\
\hline $\mathrm{C}_{7} \mathrm{H}_{15} \mathrm{CONH}\left(\mathrm{CH}_{2}\right)_{n} \mathrm{NHCOC}{ }_{7} \mathrm{H}_{15} \mathrm{k}$ & $\mathrm{C}_{7} \mathrm{H}_{15} \mathrm{CONH}\left(\mathrm{CH}_{2}\right)_{2} \mathrm{NHCOC}_{7} \mathrm{H}_{15}$ & 446 & $\mathrm{e}$ & 0.588 & 7.72 & 0.978 & 8.6 & \\
\hline trans-4-n-alkoxy-3-chlorocinnamic aci & trans-4-n & 479.2 & 0 & & 6. & 0.991 & 4.1 & \\
\hline$i d s^{b, l}$ & Cidr & 487.7 & 0 & 0.492 & 4.04 & 0.886 & 4.2 & \\
\hline nnamic acids ${ }^{b, l}$ & omocinnamic acids & 487.7 & 0 & 1.4 & 1.10 & 0.977 & 2.0 & \\
\hline cholesterol thioalkanoates ${ }^{m}$ & cholesterol thiopropionate & 385 & & 0.346 & 5.22 & 0.982 & 2.1 & 7 \\
\hline
\end{tabular}

a All compounds in these series were fit by using $380 \mathrm{~K}$ for $\mathrm{T}_{f \infty}$ unless noted otherwise; $\mathrm{S}$, even (e) and odd (o) series of $\mathrm{n}$; $\mathrm{r}^{2}$, correlation coefficient; $\pm \sigma$, standard deviation; $\mathrm{n}_{T}$, total number of data points. ${ }^{b}$ Some of the members of this series form liquid crystals. ${ }^{c}$ Reference 33. ${ }^{d}$ Reference 35. e Reference 36. ${ }^{\mathrm{f}}$ Reference 37. ${ }^{\mathrm{g}}$ Reference 22. $\mathrm{h}$ Reference 39. i Reference 38. $\mathrm{j}$ Reference $27 .{ }^{\mathrm{k}}$ Reference $21 .{ }^{\mathrm{I}}$ Reference 34. $\mathrm{m}$ Most members of this series form cholesteric phases; melting temperatures were model ed using a value of 324 for $\mathrm{T}_{\text {f }}$; the even and odd series were modeled together; the results obtained for these thioesters are not included in the statistics reported in the text; ref 39.

\section{Sources of Data}

The melting data used in this study were obtained from a variety of sources. The major sources of data for each series reported are cited in the footnotes of Tables 1-3. A supplementary table is also available. Melting temperatures for a few individual compounds have been obtained from texbooks ${ }^{13}$ and some of the commercial catalogs. ${ }^{14,15}$ The TRC Thermodynamic Tables ${ }^{16}$ also provided a significant amount of experimental melting data. A reviewer has pointed out that some of the information in the TRC Thermodynamic Tables, specifically melting temperatures for compounds containing between 20 and 40 carbon atoms 
Table 3. Melting-Structure Correlations of Series Related to Other Polymers ${ }^{\mathrm{a}}$

\begin{tabular}{|c|c|c|c|c|c|c|c|c|}
\hline homologous series & parent compound & $\begin{array}{c}\mathrm{T}_{\mathrm{f}} / \mathrm{K} \\
\text { parent }\end{array}$ & $\mathrm{S}$ & $\mathrm{m}$ & $b$ & $r^{2}$ & $\pm \sigma / \mathrm{K}$ & $\mathrm{n}_{\mathrm{T}}$ \\
\hline n-perfluoroalkanes ${ }^{b, c}$ & $\begin{array}{l}\text { perfluorobutane } \\
\text { perfluoropropane }\end{array}$ & $\begin{array}{l}164 \\
125.5\end{array}$ & $\begin{array}{l}\mathrm{e} \\
\mathrm{o}\end{array}$ & $\begin{array}{l}0.159 \\
0.140\end{array}$ & $\begin{array}{l}0.768 \\
0.855\end{array}$ & $\begin{array}{l}0.999 \\
0.920\end{array}$ & $\begin{array}{r} \pm 1.3 \\
\pm 14.3\end{array}$ & $\begin{array}{l}6 \\
4\end{array}$ \\
\hline $\mathrm{H}\left[\mathrm{OCH}_{2} \mathrm{CH}_{2}\right]_{n} \mathrm{OH}^{\mathrm{b}}$ & $\begin{array}{l}\text { Polyoxyethylene }\left(\mathrm{T}_{\mathrm{f}}=342 \mathrm{~K}\right) \\
\mathrm{H}\left[\mathrm{OCH}_{2} \mathrm{CH}_{2}\right]_{2} \mathrm{OH} \\
\mathrm{H}\left[\mathrm{OCH}_{2} \mathrm{CH}_{2}\right] \mathrm{OH}\end{array}$ & $\begin{array}{l}267.2 \\
260.6\end{array}$ & $\begin{array}{l}\mathrm{e} \\
\mathrm{o}\end{array}$ & $\begin{array}{l}0.407 \\
0.554\end{array}$ & $\begin{array}{l}3.36 \\
2.34\end{array}$ & $\begin{array}{l}0.884 \\
0.953\end{array}$ & $\begin{array}{l} \pm 4.7 \\
\pm 5.2\end{array}$ & $\begin{array}{l}8 \\
8\end{array}$ \\
\hline $\mathrm{CH}_{3} \mathrm{CO}\left(\mathrm{OCH}_{2}\right)_{n} \mathrm{O}_{2} \mathrm{CCH}_{3}{ }^{\mathrm{b}}$ & $\begin{array}{l}\text { Polyoxymethylene }\left(T_{f}=473.2 \mathrm{~K}\right) \\
\text { 1,3-diacetoxy-2-oxapropane } \\
\text { diacetoxymethane }\end{array}$ & $\begin{array}{l}260.2 \\
250.2\end{array}$ & $\begin{array}{l}\mathrm{e} \\
\mathrm{o}\end{array}$ & $\begin{array}{l}0.205 \\
0.171\end{array}$ & $\begin{array}{l}1.19 \\
1.66\end{array}$ & $\begin{array}{l}0.998 \\
0.969\end{array}$ & $\begin{array}{l} \pm 9.1 \\
\pm 7.8\end{array}$ & $\begin{array}{l}8 \\
7\end{array}$ \\
\hline $\begin{array}{l}\mathrm{H}\left[\mathrm{NH}\left(\mathrm{CH}_{2}\right)_{5} \mathrm{CO}\right]_{n} \mathrm{OH}^{b} \\
\mathrm{C}_{2} \mathrm{H}_{5} \mathrm{CO}\left[\mathrm{NH}\left(\mathrm{CH}_{2}\right)_{5} \mathrm{CO}\right]_{n} \mathrm{NHC}_{3} \mathrm{H}_{7}{ }^{b}\end{array}$ & $\begin{array}{l}\text { Nylon-6 }\left(\mathrm{T}_{\mathrm{f}}=533 \mathrm{~K}\right) \\
\text { [ } \epsilon \text {-aminocaproic acid] }]_{2} \\
\epsilon \text {-aminocaproic acid } \\
\mathrm{C}_{2} \mathrm{H}_{5} \mathrm{CO}\left[\mathrm{NH}\left(\mathrm{CH}_{2}\right)_{5} \mathrm{CO}\right]_{3} \mathrm{NHC}_{3} \mathrm{H}_{7} \\
\mathrm{C}_{2} \mathrm{H}_{5} \mathrm{CO}\left[\mathrm{NH}\left(\mathrm{CH}_{2}\right)_{5} \mathrm{CO}\right]_{2} \mathrm{NHC}_{3} \mathrm{H}_{7}\end{array}$ & $\begin{array}{l}471.2 \\
479.2 \\
422.8 \\
379.2\end{array}$ & $\begin{array}{l}\mathrm{e} \\
\mathrm{o} \\
\mathrm{e} \\
\mathrm{o}\end{array}$ & $\begin{array}{l}0.089 \\
0.046 \\
0.194 \\
0.236\end{array}$ & $\begin{array}{r}10.0 \\
9.90 \\
6.97 \\
6.51\end{array}$ & $\begin{array}{l}0.650 \\
0.599 \\
0.919 \\
0.769\end{array}$ & $\begin{array}{l} \pm 0.8 \\
\pm 1.6 \\
\pm 1.9 \\
\pm 2.7\end{array}$ & $\begin{array}{r}5 \\
10 \\
7 \\
6\end{array}$ \\
\hline $\begin{array}{l}\mathrm{CH}_{3} \mathrm{O}_{2} \mathrm{CC}_{6} \mathrm{H}_{4} \mathrm{CO}- \\
\quad\left[\mathrm{OCH}_{2} \mathrm{CH}_{2} \mathrm{O}_{2} \mathrm{CC}_{6} \mathrm{H}_{4} \mathrm{CO}\right]_{n} \mathrm{OCH}_{3} \mathrm{~b}\end{array}$ & $\begin{array}{l}\text { Poly(ethylene terephthalate) }\left(\mathrm{T}_{\mathrm{f}}=543.2\right. \\
\mathrm{CH}_{3} \mathrm{O}_{2} \mathrm{CC}_{6} \mathrm{H}_{4} \mathrm{CO}\left[\mathrm{CCH}_{2} \mathrm{CH}_{2} \mathrm{O}_{2} \mathrm{CC}_{6} \mathrm{H}_{4} \mathrm{CO}_{2} \mathrm{OCCH}_{3}\right. \\
\mathrm{CH}_{3} \mathrm{O}_{2} \mathrm{CC}_{6} \mathrm{H}_{4} \mathrm{CO}_{2} \mathrm{CH}_{2} \mathrm{CH}_{2} \mathrm{O}_{2} \mathrm{CC}_{6} \mathrm{H}_{4} \mathrm{CO}_{2} \mathrm{CH}_{3}\end{array}$ & $\begin{array}{l}\text { K) } \\
467.2 \\
441.2\end{array}$ & $\begin{array}{l}\mathrm{e} \\
\mathrm{o}\end{array}$ & $\begin{array}{l}2.24 \\
3.37\end{array}$ & $\begin{array}{l}11.1 \\
2.70\end{array}$ & $\begin{array}{l}0.992 \\
0.987\end{array}$ & $\begin{array}{l} \pm 6.0 \\
\pm 5.4\end{array}$ & $\begin{array}{l}4 \\
4\end{array}$ \\
\hline
\end{tabular}

a Melting temperatures calculated using eqs 4 and 5 unless otherwise noted; S, even (e) and odd (o) series of $n$; $r^{2}$, correlation coefficient; $\pm \sigma$, standard deviation; $\mathrm{n}_{\mathrm{T}}$, total number of data points. ${ }^{\mathrm{b}}$ Reference 23 . c Reference 40 .

in the alkyl portion of the molecule, may not be experimental. Data taken from these tables in this carbon range were not used unless referenced to the original literature. It should also be noted that there are wide discrepancies in the uncertainties associated with the data set. The nonlinear least-squares calculations described below are unweighted, and it should not be expected that melting temperatures calculated in this way lie within the uncertainty limits of the better data.

\section{Modeling the Melting Temperatures of a Homologous Series}

The model that is proposed to estimate the melting of a homologous series of compounds is derived from observations of the melting behavior of the n-alkanes. The data reported in Figure 1 summarize the behavior observed for the even n-alkanes. A similar relationship is observed for the odd numbered n-alkanes as well (graph not shown). This odd-even alternation in melting behavior and in other physical properties in the n-alkanes is well-known. The segregation of compounds into even and odd numbered carbon chains is the first step in the protocol that is described below. The model we propose assumes that the melting behavior of any homologous series will mimic the curve shown in Figure 1 and will hyperbolically approach the melting temperature of the polymer that the series most closely resembles. This applies to any homologous series of molecules characterized by the same symmetry number. In the case of the n-alkanes, the melting should approach the melting temperature of polyethylene, $\mathrm{T}_{\mathrm{f}}(\infty)$ $=415 \mathrm{~K}$, as the limiting value. Melting data of monomers and oligomers related to other polymers are more limited, but the data available for polyfluorinated alkanes, a limited series of polyoxyalkanes, polyalkoxyesters, and polyamides related to Nylon 6, appear to support this behavior. This is discussed below in more detail.

A. Series Related to Polyethylene with Parents Melting bel ow 411.3 K. The hyperbolic behavior observed in the melting of the even n-alkanes in Figure 1 can be modeled successfully by noting that if the following function

$$
1 /\left[1-\mathrm{T}_{\mathrm{f}}(\mathrm{n}) / \mathrm{T}_{\mathrm{f}}(\infty)\right]
$$

is plotted against the number of methylene groups (n), a

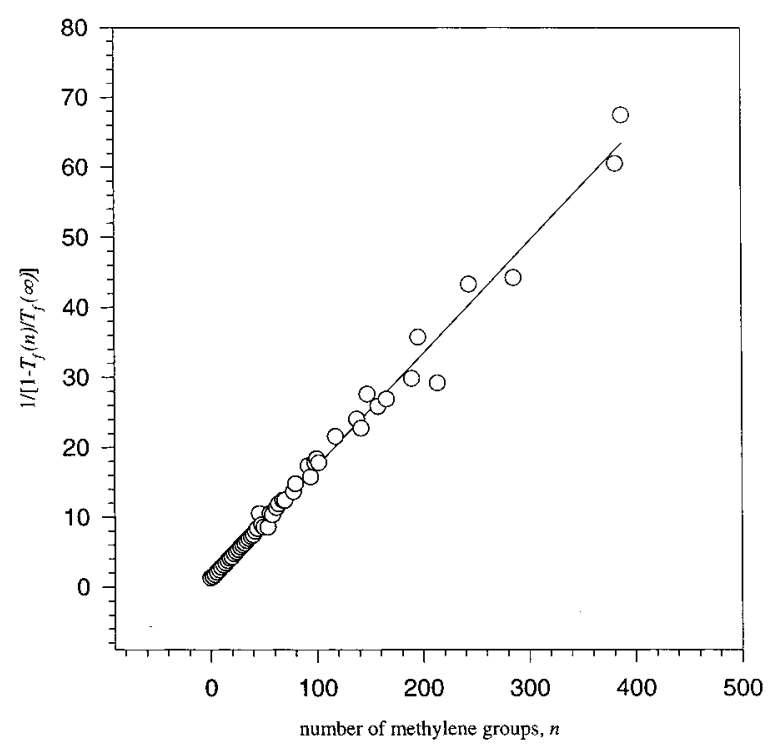

Figure 2. Correlation between the function $1 /\left[1-T_{f}(n) / T_{f}(\infty)\right]$ and the number of methylene groups for the even $n$-alkanes. The terms $T_{f}(n)$ and $T_{f}(\infty)$ represent the melting point of the compound with $n$ methylene groups and the melting point of polyethylene, respectively.

straight line is obtained as shown in Figure 2 for the compounds of Figure 1 . The terms $T_{f}(n)$ and $T_{f}(\infty)$ in this function represent the melting temperature of the compound with an even number of methylene groups and the melting temperature of the crystalline polymer, polyethylene, respectively. Similar behavior characterized by a different slope and intercept is observed for the n-alkanes containing an odd number of methylene groups. This linear behavior defines an analytical relationship between the melting of each member of a series $T_{f}(n)$, the melting of the related polymer $T_{f}(\infty)$, and the number of repeat units of the oligomer $\mathrm{n}$. This relationship is expressed as eq 1 ,

$$
T_{f}(n)=T_{f}(\infty)[1-1 /(m n+b)]
$$

where the $\mathrm{m}$ and $\mathrm{b}$ terms represent the slope and intercept of the straight line, respectively.

The variables $m$ and $b$ for each correlation described bel ow were obtained by a nonlinear least-squares fit of the 


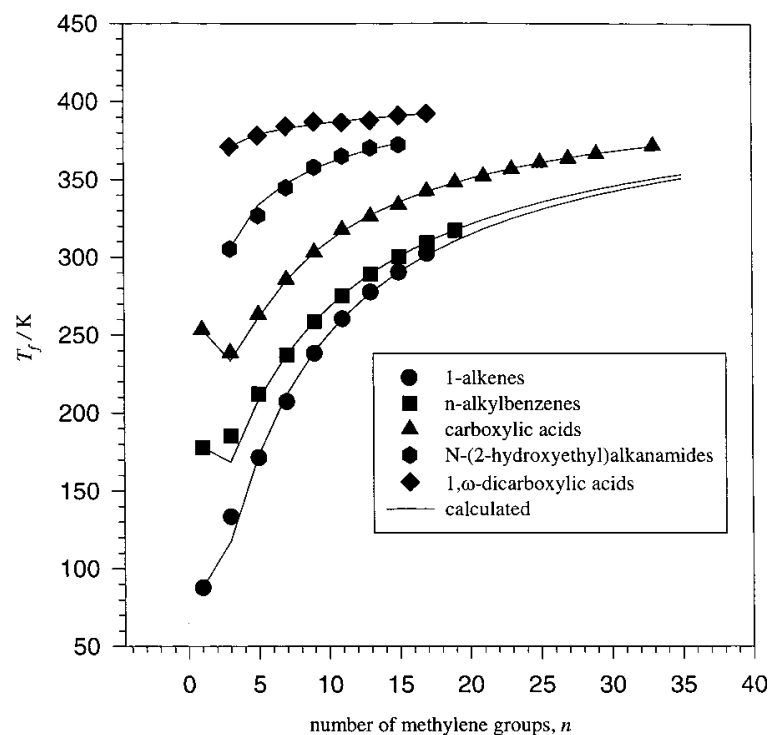

Figure 3. Melting temperatures of the odd 1-alkenes, n-alkylbenzenes, n-carboxylic acids, $\mathrm{N}$-(2-hydroxyethyl)alkanamides, and 1, $\omega$-dicarboxylic acids versus the number of methylene groups: (circles, squares, triangles, and hexagons) experimental data; (lines) calculated results.

melting temperature data as a function of the number of repeat units according to eq 1 . In the initial fitting of the data in Figure 1, the quality of the fit was found to be sensitive to the choice of $T_{f}(\infty)$ that was used. The value found to give the best fit to the even n-alkane data was $411.3 \mathrm{~K}$. This value was derived from the melting behavior of the even n-alkanes by minimizing the error observed between calculated and experimental values. It has been used in all subsequent calculations calling for the melting temperature of polyethylene unless noted otherwise. The solid line of Figure 1 was calculated with the aid of eq 1 . Values for the variables $m$ and $b$ obtained by the nonlinear least-squares fit are provided in the fifth and sixth col umn of Table 1.

Parent compounds are defined for many series as the compound with either one (odd series) or two methylene groups (even series, Table $\overline{1}$ ); in a more general context they are defined as the compound with either one (o) or two repeat units (e). Unlike the even n-alkanes of Figure 2 , some initial curvature from a straight line is generally observed in the plot of $1 /\left(1-T_{f}(n) / T_{f}(\infty)\right)$ against $n$ for the first two members of many homologous series. Curvature in both directions from the straight line has been observed. This curvature may become more pronounced as the melting temperature of the parent compound of a series increases. As a consequence of this curvature, the melting temperatures of the parent and the first member of both the even and odd numbered series have been excluded from all the nonlinear regression analyses used to calculate the variables $m$ and $b$. Figure 3 illustrates the typical melting behavior observed for various series characterized by parent compounds melting below $411.3 \mathrm{~K}$. The symbols represent experimental data while the lines are calculated from the nonlinear regression analysis.

The results of fitting eq 1 to a wide variety of different homol ogous series with parents melting below $411.3 \mathrm{~K}$ are given in Table 1. The generic name of the series is provided in the first column of the table. This is followed by the name of the first available member of the series in col umn 2; the corresponding melting temperature of this material is provided in the third column. The fourth column defines the nature of the series as either even (e) or odd (o) on the basis of the number of repeat units, $n$. The fifth, sixth, and seventh columns provide a description of the variables $m$ and $b$ and the correlation coefficient $\left(r^{2}\right)$ derived from the nonlinear least-squares fit. The standard deviation associated with the differences between experimental and calculated results and the total number of data points, $n_{T}$, used to establish each correlation are listed in the last two columns, respectively. Parent compounds melting close to $411.3 \mathrm{~K}$ are discussed below.

As noted above, curvature is often observed in the first few members of each series. In an effort to correct for this curvature, a second function, selected to reproduce the melting temperature of the parent and then decay exponentially as a function of n, was used to provide some correction to the first few members of each series. The function $f(n)$ is defined as

$$
f(n)=A_{0}\left(\left|\Delta A_{0}\right| / A_{0}\right)^{n / n_{0}}
$$

where

$\Delta \mathrm{A}_{0}=\mathrm{A}_{0^{\prime} \text { expt }}-\mathrm{A}_{0^{\prime} \text { calc }}=1 /\left[1-\mathrm{T}_{\mathrm{f}}\left(\mathrm{n}_{0}\right) / \mathrm{T}_{\mathrm{f}}(\infty)\right]-\left(\mathrm{mn}_{0}+\mathrm{b}\right)$

and $n_{0}$ represents the number of repeat groups of the parent of the series and $\left|\Delta A_{0}\right|$ represents the absolute value of the difference between $A_{0}$ calculated from the experimental melting of the parent, $A_{0, \text { expt, }}$ and $A_{0}$ calculated from the correlation, $A_{0, \text { calc }}($ eq 3$)$. The function $f(n)$ was designed to decrease rapidly with increasing values of $n$. The melting of the remaining members of the series was then calculated from eq 1, adjusted as follows:

$$
T_{f}(n)=T_{f}(\infty)[1-1 /(m n+b+f(n))] \text { if } \quad \Delta A_{0}>0
$$

or

$$
T_{f}(n)=T_{f}(\infty)[1-1 /(m n+b-f(n))] \text { if } \quad \Delta A_{0}<0
$$

In instances when $\Delta A_{0}$ was positive, $f(n)$ was added to the value of $m n+b$ (eq 4), and in cases when the term was negative, the value was subtracted from it (eq 5). The solid lines shown in Figure 3 were calculated using eqs 4 and 5 . Equations 4 and 5 were used in reproducing the melting temperatures of all the parent compounds listed in Table 1 melting up to $390 \mathrm{~K}$, unless indicated otherwise in the tables. Their use usually resulted in an improvement in the fit of the first few members of the series. If the melting temperature of the parent compound in the series is unknown, any compound can be chosen as the parent. In these instances the $\Delta \mathrm{A}_{0}$ correction is usually small and can be neglected. Equation 4 or 5 was not used in a few cases when the function, $f(n)$, did not decay exponentially with increasing $\mathrm{n}$. In these cases the parent compound was excluded from all correlations and no corrections were applied.

Prediction of the melting temperatures of series with parents melting near $411 \mathrm{~K}$ is problematic. Equations 1 , 4 , and 5 are not well behaved near their asymptotes. In most of these cases the experimental melting temperature first drops below $411 \mathrm{~K}$ and then begins a gradual ascent toward this value. The melting of a limited number of the even series of $\mathrm{N}$-( $\beta$-naphthyl)alkanamides and the odd series of $1, \omega$-alkanedioic acids listed in Table 1 was model ed using eq 1 in the following manner. The melting temperatures of the first few members of each series were ignored until the melting temperatures began to increase 


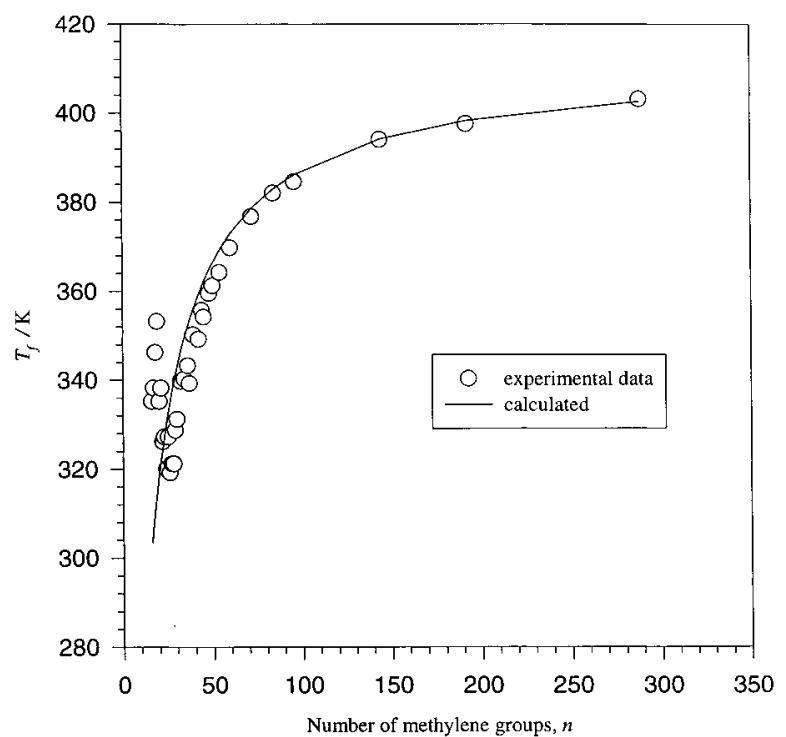

Figure 4. Melting temperatures of the cycloalkanes versus the number of methylene groups. Both even and odd members are included.

as a function of $n$. Equation 1 was then used to model melting of the remaining members of the series.

Table 1 has been divided into sections in order to highlight several features of the correlations observed. Section A provides a summary of the results obtained for the hydrocarbon series examined. The fit obtained for all these series is quite good. The results suggest that, regardless of the type of alkyl substitution or its location, eqs 1,4 , and 5 will model the melting of the corresponding homologous series effectively. In all these cases, the correlations are applicable to compounds with the same symmetry number. ${ }^{17}$ Currently, there are no examples of series with $1, \omega$-dialkyl substitution; results obtained with other functional groups suggest that these equations will also work here as well.

The results observed for cycloalkanes are summarized in Table 1B. With the exception of a few of the small to medium sized rings, cycloalkanes surprisingly show a reasonably good correlation. A plot of the experimental melting temperatures versus the number of methylene groups is shown in Figure 4. In this case both the even and odd series were combined. The solid line represents the calculated value using eq 1 ; the variables $m$ and $b$ for this fit are given in Table 1. Apparently, as the ring becomes large enough, the methylene groups are sufficiently flexible to mimic the behavior of their acyclic analogues. The cycloalkanes, however, give the poorest correlation of any series tested. This is mainly a consequence of melting anomalies observed in the small to medium sized rings. The symmetry numbers of members of this series vary. Various reasons for these anomalies are discussed below.

The results obtained for alkanes functionalized at terminal positions with a variety of different functional groups are summarized in Table 1C. The melting of these compounds is fit using eqs 4 and 5 . Most of these compounds are substituted only at one terminal position. As is suggested by the results provided by the 1 , $\omega$-di hydroxyalkanes, the last two entries in this section of the table, eqs 1, 4, and 5 also appear applicable in describing the melting behavior of $1, \omega$-disubstitution. It should al so be noted that polysubstituted compounds such as $\mathrm{F}\left[\mathrm{CF}_{2}\right]_{12}\left[\mathrm{CH}_{2}\right]_{n} \mathrm{H}$, where $\mathrm{n}$ is the variable, correlate using the melting temperature

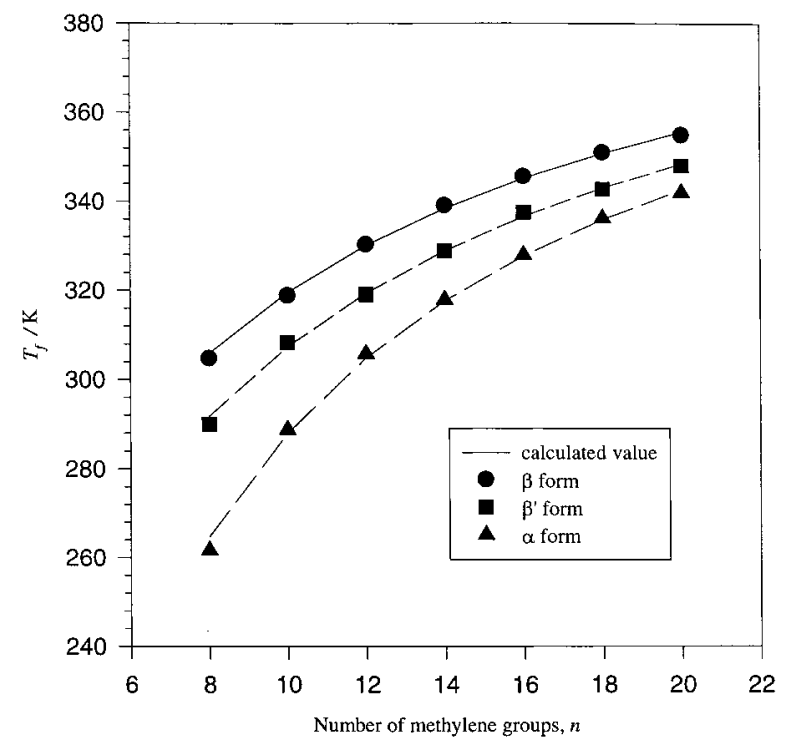

Figure 5. Experimental melting points of the three polymorphic forms of symmetric glycerol trialkanoates ranging from decanoate to eicosanoate. Molecular packing in each series is very similar.

of polyethylene. While melting data for a sufficient number of compounds such as $F\left[\mathrm{CF}_{2}\right]_{n}\left[\mathrm{CH}_{2}\right]_{12} \mathrm{H}$ are not apparently available, we would expect such compounds to correl ate to the melting temperature of Teflon as discussed below.

Section D of Table 1 summarizes the results obtained by examining symmetrically di- and trisubstituted functional groups. The reasonably good correlations observed for symmetrically substituted ethers, secondary amines, and particularly tertiary amines and symmetrically substituted 1,3-di- and 1,2,3-triglycerides, in view of the presence of branching, were surprising and somewhat unexpected. These results suggest that the melting behavior of both symmetrically and unsymmetrically branched series could be correlated, provided the size of the groups was varied in a uniform manner. The correlations observed for the even series of symmetrically substituted saturated triglycerides provide some additional insight into the origin of the correlations observed. The sym-triglycerides containing fatty acids with 10 to 22 carbons each crystallize in three different polymorphic forms, an $\alpha$ form, a $\beta^{\prime}$ form, and a $\beta$ form; the latter is the most stable thermodynamically. ${ }^{18}$ The $\alpha$ form of each member of the series is favored kinetically and forms a loosely packed hexagonal crystal structure. The $\beta^{\prime}$ form (of intermediate stability) crystallizes in an orthorhombic structure, and the most stable form, characterized by the highest melting temperature, crystallizes in a triclinic crystal structure. Melting of the polymorphs of each series is successfully model ed by eq 1 , as illustrated in Figure 5. Each polymorphic form packs similarly in the crystal, suggesting that si milarity in crystal packing may be an important structural factor responsible for the correlations observed. Other even and/or odd series are known to show similar packing behavior within each series. These include the n-alkanes and some of the other series listed as functionalized alkanes in Table $1 .{ }^{19}$ Differences in packing between members of a series may also be responsible for the anomalous melting behavior observed by some of the small to medium sized cycloalkanes.

A total of 832 compounds were used to generate the slopes and intercepts included in Table 1. The standard deviation associated with differences between experimental and calculated melting temperatures of each series excluding the parent is provided in the eighth column of Table 1 
for each entry. Even with the $f(n)$ correction, the standard deviation was usually dominated by the uncertainty in the fit of the first few members of the series. The last column of Table 1 provides the number of data points available for each series. If all of the compounds are included in the correlation, the fit was characterized by a standard deviation of $\pm 6.1 \mathrm{~K}$. Removal of the cycloalkanes from the database reduces the standard deviation of the remaining 796 compounds to $\pm 4.1 \mathrm{~K}$.

B. Series Related to Polyethylene with Parents Melting above $411.3 \mathrm{~K}$. According to the model proposed above, in cases where the melting temperature of the parent exceeds the melting temperature of polyethylene, melting of each member of the series should decrease as the number of repeat units, $n$, increases. In these cases, the melting temperature should continue to decrease systematically, approaching the value of $411.3 \mathrm{~K}$ asymptotically. I deally, the melting behavior of such a series should bear a mirror image resemblance to the behavior observed for the n-alkanes. Melting of these compounds should therefore be modeled by a decreasing hyperbolic function, adjusted to asymptotically approach $411.3 \mathrm{~K}$ as a minimum.

From the limited amount of data examined, we have found series with parent compounds melting above $500 \mathrm{~K}$ can be characterized by such a function. A plot of $1 /[1-$ $\left.T_{f}(\infty) / T_{f}(n)\right]$ versus the number of methylene groups in the molecule also results in a linear correlation. The use of $411.3 \mathrm{~K}$ for $\mathrm{T}_{\mathrm{f}}(\infty)$ works well in these cases.

$$
T_{f}(n)=T_{f}(\infty) /[1-1 /(m n+b)]
$$

In some homol ogous series, particularly those characterized by parent compounds mel ting above 411 but below 500 $\mathrm{K}$, we have found that the melting temperature of several members of the series may initially drop below $411.3 \mathrm{~K}$. A limited amount of available data suggests that the melting temperature then gradually increases with increasing chain length. To avoid the problems associated with fitting these data with a hyperbolic function near (or below) its asymptotic value, we have used a value of 380 in place of $411.3 \mathrm{~K}$ for $\mathrm{T}_{\mathrm{f}}(\infty)$. This is illustrated in Figure 6 for several different systems, including some series with parents melting above $500 \mathrm{~K}$. The symbols represent experimental data, and the solid lines are those calculated by eq 6 using a value of $380 \mathrm{~K}$ for $T_{f}(\infty)$. This equation successfully models the descending portion of the melting curve for available experimental data. For those series in which the melting temperature drops below $411.3 \mathrm{~K}$, the ascending portion of the melting curve can in principle be modeled using eq 1 . However, we have not been able to locate sufficient experimental data in these systems to verify this behavior. The value of $380 \mathrm{~K}$ was chosen for $\mathrm{T}_{\mathrm{f}}(\infty)$ on the basis that this value gave a good fit to all available data exhibiting descending hyperbolic behavior. Some series with parent compounds melting near $411.3 \mathrm{~K}$ may have members in the series melting below $380 \mathrm{~K}$ and may require the use of other values for $T_{f}(\infty)$.

The series in Figure 6 include melting of the odd series of the n-alkoxybenzoic acids, the 6-n-alkoxy-2-naphthoic acids, and the 4-n-alkoxy-3-fluorobenzoic acids and the even series of 8-alkyltheophyllines. The melting temperatures of these compounds decrease with increasing chain length and appear to approach the melting of polyethylene. The melting temperatures of the 4-n-alkoxy-3-fluorobenzoic acids initially drop slightly bel ow $411.3 \mathrm{~K}$, but the melting temperature then begins to rise again with an increase in chain length.

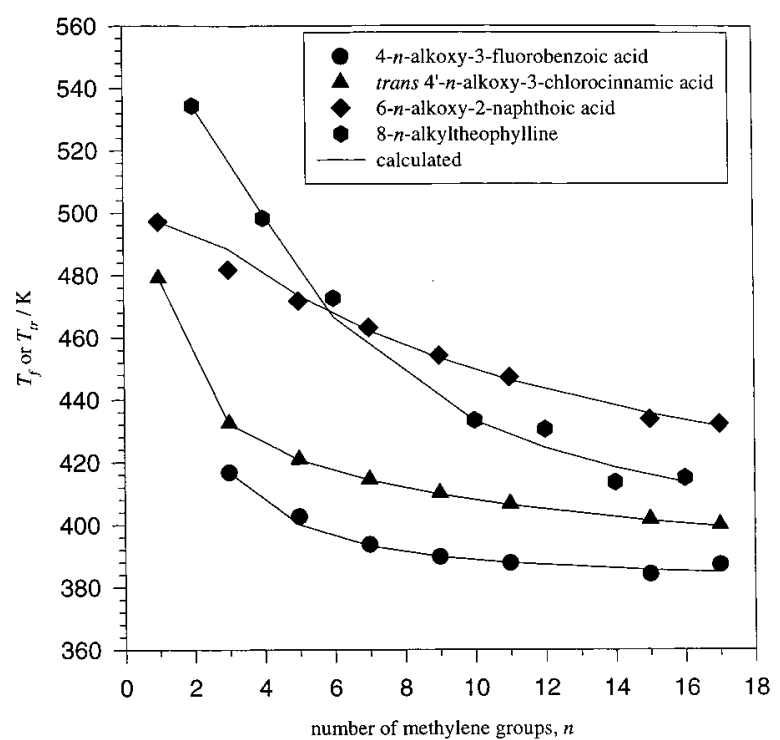

Figure 6. Experimental melting or smetic/nematic $\rightarrow$ isotropic transition temperatures for the odd series of 4-alkoxy-3-fluorobenzoic acids, trans-4'-n-alkoxy-3-chlorocinnamic acids, and 6-alkoxy2-naphthoic acids and the even series of 8-alkyltheophyllines: (symbols) experimental data; (lines) calculated results.

As observed with previous series, melting of the parent compounds in many of these series also shows some deviation from the value predicted by eq 6 . The melting temperatures of the parent and the second member of each series were not included in generating the correlations listed in Table2. Equation 6 has been modified in a fashion similar to that for eq 1 . This resulted in eqs 7 and 8 . The function $\mathrm{g}(\mathrm{n})$ was modeled to reproduce the melting temperature of the parent and then decrease exponentially with increasing $\mathrm{n}$. The series illustrated in Figure 6 and tabulated in Table 2 were calculated with the aid of eqs 7 and 8 except in a few cases where the function $\mathrm{g}(\mathrm{n})$ did not decay exponentially. In the latter case the melting temperature of the parent was not used.

$$
T_{f}(n)=T_{f}(\infty) /[1-1 /(m n+b+f(n))] \text { if } \quad \Delta B_{0}>0
$$

or

$$
T_{f}(n)=T_{f}(\infty) /[1-1 /(m n+b-f(n))] \quad \text { if } \quad \Delta B_{0}<0
$$

where

$$
g(n)=B_{0}\left(\left|\Delta B_{0}\right| / B_{0}\right)^{n / n_{0}}
$$

and

$\Delta \mathrm{B}_{0}=\mathrm{B}_{0, \text { expt }}-\mathrm{B}_{0, \text { calc }}=1 /\left[1-\mathrm{T}_{\mathrm{f}}(\infty) / \mathrm{T}_{\mathrm{f}}\left(\mathrm{n}_{0}\right)\right]-\left(\mathrm{mn}_{0}+\mathrm{b}\right)$

A summary of the results obtained examining various series with parents melting above $411 \mathrm{~K}$ is given in Table 2. The data are presented in the same format used in Table 1. Melting temperatures were cal culated for all compounds using a value of $380 \mathrm{~K}$ for $\mathrm{T}_{\mathrm{f}}(\infty)$. A total of 113 compounds, excluding the parents of each series, were included in the correlations. The standard deviation of each fit and the number of data points used to establish the correlation in each series are given in the last two columns of Table 3. The standard deviation between experimental and calculated melting points for the 113 compounds in the database was $\pm 2.6 \mathrm{~K}$. 


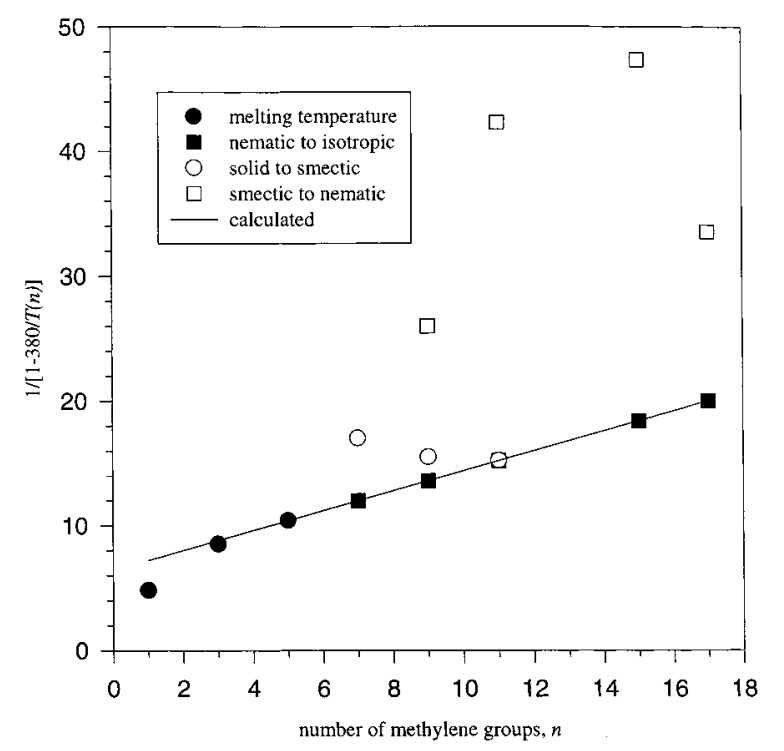

Figure 7. Plot of $1 /[1-T(\infty) / T(n)]$ verus the number of methylene groups for trans-4-n-alkoxy-3-chlorocinnamic acids. The solid circles represent melting temperatures, the solid squares represent nematic to isotropic transitions, the circles represent solid to smectic transitions, and the squares represent smectic to nematic transitions. The temperatures at which the liquids become isotropic appear to correlate best. A value of $380 \mathrm{~K}$ was used for $\mathrm{T}(\infty)$.

The series listed in Table 2 differ from those in Table 1 in two significant ways. First, there is clearly far less experimental data available for each series. A second significant difference is that many of the compounds in some series form liquid crystals. Only the first few members of the series melt directly to the isotropic liquid state. Liquid crystalline behavior is frequently encountered as the size of the alkyl group increases. M ost liquid crystals are characterized by several transition temperatures in going from solid to liquid (e.g. solid-smectic, smecticnematic, nematic-isotropic). Do normal melting temperatures correlate with any of these transitions? In the examples we have examined, the temperature at which the liquid crystal becomes isotropic correlates best with the other members of the series. This is illustrated in Figure 7 for several trans-4-n-alkoxy-3-chlorocinnamic acids. ${ }^{20}$ The first three members of this series melt directly to the isotropic liquid. The remaining compounds exhibit various transitions. In this case it is the nematic to isotropic transition that correlates best with the normal melting temperatures. This behavior is typical of most of the series examined thus far with members forming liquid crystals.

According to the model proposed above, as the size of the alkyl chain increases, the melting behavior of any molecule should gradually approach the melting temperature of polyethylene. What influence does the size of the molecule have on the temperature at which melting becomes dominated by the alkyl side chain? Examination of the behavior of a series of thiocholesteryl alkanoates ${ }^{21}$ suggests that, for large mol ecules, the side chain might also have to be quite large before it exerts a controlling influence over the melting temperature. Most, but not all, of the thiochol esteryl al kanoates form cholesteric phases. Transition temperatures to the isotropic liquid are shown in Figure 8. In this case, the temperatures for both the odd and even series were model ed using the same parameters in eq 8. The first two members of the even and odd series were not used in the correlation; the melting of thiocholesterol propanoate was used to generate the $\mathrm{g}(\mathrm{n})$ term. A

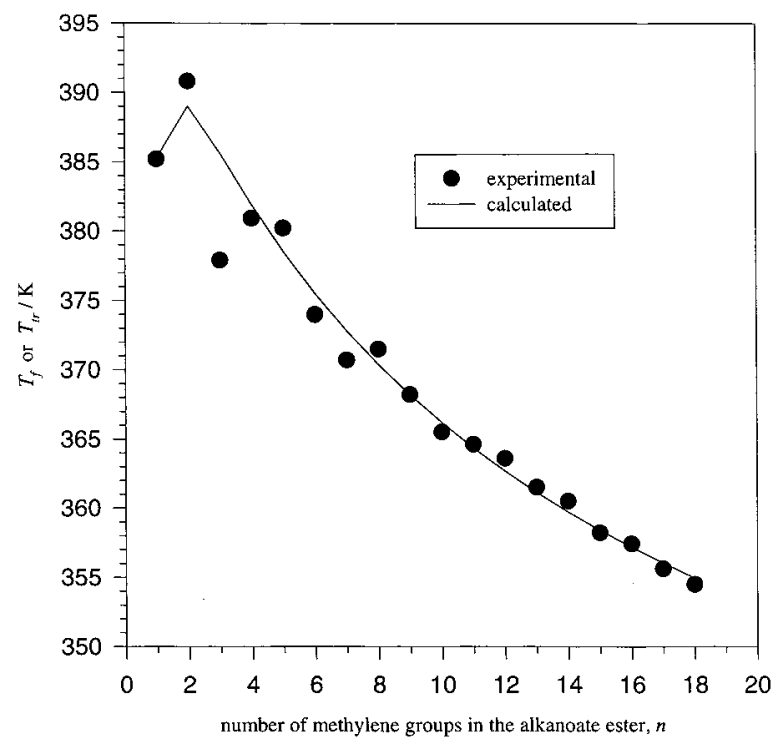

Figure 8. Melting $\left(T_{f}\right)$ or cholesteric-isotropic transition temperatures $\left(T_{t r}\right)$ for both the even and odd members of a series of thiocholesterol alkanoates as a function of the number of methylene groups in the alkanoate side chain. The line was calculated using a value of $324 \mathrm{~K}$ for $\mathrm{T}(\infty)$ in eq 8 .

nonlinear least-squares fit to the experimental data suggests a value of $324 \mathrm{~K}$ for $\mathrm{T}_{\mathrm{f}}(\infty)$. The parameters derived from this correlation are listed at the bottom of Table 2 . This temperature is considerably lower than the $380 \mathrm{~K}$ used to fit the series listed in Table 2 and may be a reflection of the effect of the size of the steroidal portion of the molecule. If the alkyl side chain is increased further, some point should be reached when the temperature will start to increase and begin to approach $411 \mathrm{~K}$. Likewise, according to the model proposed, at some point the esters should no longer form cholesteric phases. The results obtained for these compounds are not included in any of the statistics reported below.

C. Modeling Melting Temperatures of Series Related to Other Polymers. In principle, eqs 1-8 should be capable of predicting the effect of chain length on melting of other linear molecules provided the melting temperature of the polymer is available. The amount of data we have been able to retrieve from the literature for ol igomers related to other polymers is limited. Experimental melting temperature data for several ol igomeric systems related to the following polymers have been retrieved: Teflon $\left(T_{f}(\infty)=605 \mathrm{~K}\right)$, polyoxymethylene $\left(T_{f}(\infty)=473 \mathrm{~K}\right)$, polyoxyethylene $\left(T_{f}(\infty)=342 \mathrm{~K}\right)$, Nylon-6 (poly $(\epsilon$-aminocaproic acid) $\left.\left(T_{f}(\infty)=533 \mathrm{~K}\right)\right)$, and poly(ethylene terephthalate) $\left(T_{f}(\infty)=543.2 \mathrm{~K}\right)$. In all of these cases, the oligomers melt below the melting temperature of the polymer. Consequently, only eqs 4 and 5 have been used.

The results obtained with these polymers are listed in Table 3. Data for nine different series related to five different polymers and comprising a total of 95 compounds were retrieved from the literature. The parameters generated are listed in Table 3. Melting of the even series of polyfluorinated compounds related to Teflon is shown as solid circles in Figure 9. This figure also illustrates the results observed in the melting behavior of a series $1, \omega$ dihydroxyethyl ethers related to polyoxyethylene and $\epsilon$-aminocaproic acids related to Nylon-6. Excluding the parents, the melting temperatures of the 95 oligomers correlated by the parameters listed in Table 3 were reproduced with a standard deviation of $\pm 5.7 \mathrm{~K}$. 


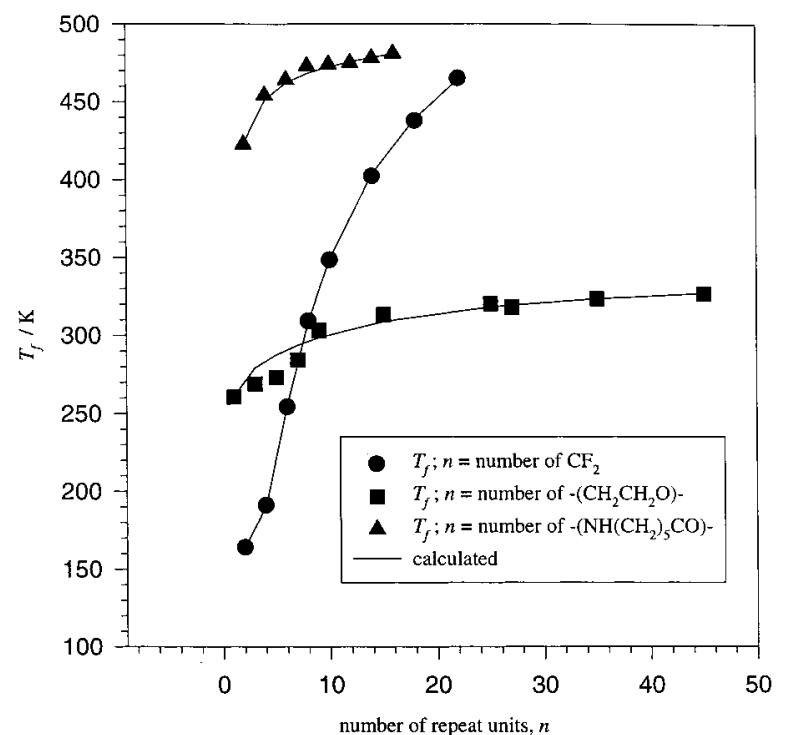

Figure 9. Experimental melting points as a function of the number of repeat units: (circles) perfluoro-n-alkanes; (squares) $\mathrm{H}\left[\mathrm{OCH}_{2} \mathrm{CH}_{2}\right]_{n} \mathrm{OH}$; (triangles) $\mathrm{C}_{2} \mathrm{H}_{5} \mathrm{CO}\left[\mathrm{NH}\left(\mathrm{CH}_{2}\right)_{5} \mathrm{CO}\right]_{n} \mathrm{NHC}_{3} \mathrm{H}_{7}$.

D. Statistical Summary. The melting temperatures of a total of 1045 compounds were correlated using the parameters reported in Tables $1-3$. This resulted in a standard deviation of $\pm 5.8 \mathrm{~K}$, overall. This excludes parent compounds used in conjunction with eqs $4-8$, and the cholesteric esters. A standard deviation of $\pm 21 \mathrm{~K}$ was associated with the cycloalkanes. Excluding these compounds from consideration reduces the standard deviation to $\pm 4.1 \mathrm{~K}$ for 1004 compounds.

\section{Estimating Melting Temperatures of Homologous Series}

The correlations observed in Tables $1-3$ using eqs 1-8 suggest a simple protocol that can be followed whenever the melting temperatures of a few members of a series are available. To test how well such a protocol would reproduce the melting temperatures of the 1004 compounds of this study, we chose three compounds from each of these series and used their melting temperatures to predict the values for the remaining members of the series. The three members of each even and odd series were chosen using the following guidelines. The first two members of each homologous series were excluded. For those compounds with parents that melted below the melting of the related polymer, representatives were chosen whenever possible that melted at $300 \mathrm{~K}$ and above. Consecutive members of a series were chosen when available. The equation of the line was obtained from a linear regression analysis of $1 /[1$ - $T_{f}(n) / T_{f}(\infty)$ ] versus $n$ using these three points. The melting temperature of the parent was then used to adjust the melting temperatures of the remaining members of each series according to eqs 3 and 4 . This protocol used a total of four data points from each series to predict the remaining melting values of the series. Cyclic hydrocarbons were not induded. Oligomers were selected in a similar fashion. The melting temperature of each respective polymer was used for $T_{f}(\infty)$ in eqs 3 and 4.

A similar protocol was used to predict the melting behavior of those hydrocarbon derivatives with parent compounds melting above the melting temperature of polyethylene. The first two members of each series were excluded from sel ection of the data points used to establish the linear correlation. Consecutive members were chosen

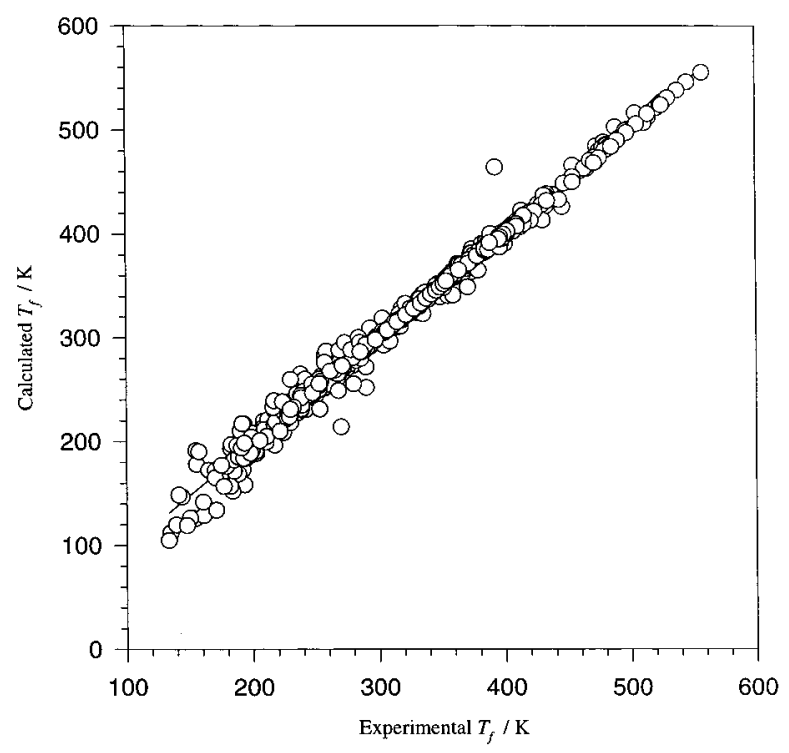

Figure 10. Comparison of 995 experimental and calculated melting temperatures.

when possible. A total of four points, including the melting of the parent, were used to establish each correlation. A value of $380 \mathrm{~K}$ was used for $T_{f}(\infty)$ in eqs 6 and 7.

This protocol was selected for several reasons. First, it takes advantage of the melting trends observed in this study. Melting temperatures above $300 \mathrm{~K}$ are also more likely to be available in the literature, as are homologous series with repeating units that vary in consecutive fashion; the latter are also most likely to produce the largest uncertainty in the two parameters $\mathrm{m}$ and $\mathrm{b}$. In these estimations, a linear regression analysis was used to evaluate $m$ and $b$. The values were evaluated from plots of $n$ versus either $1 /\left[1-T_{f}(n) / T_{f}(\infty)\right]$ or $1 /\left[1-T_{f}(\infty) / T_{f}(n)\right]$ depending on the melting point behavior of the series. A linear regression analysis provides both a visual and numerical assessment of the linearity of the data, and this can be an advantage in assessing the quality of the estimation. In a few cases, the three points plotted were severely nonlinear; a different third experimental data point was chosen in these cases. Since the temperatures chosen are usually far removed from the asymptotic limit, very little numerical differences are observed between the linear and nonlinear least-squares methods. These guidelines should provide a realistic assessment of the error associated when applying these estimations to systems with a limited amount of available experimental data.

The results of these calculations are summarized in Figure 10. The correlation coefficient $\left(r^{2}\right)$, slope, and intercept of the line generated by the data were given as

$\mathrm{T}_{\mathrm{f}, \mathrm{calc}}=(1.01 \pm 0.003) \mathrm{T}_{\mathrm{f}, \exp }-(3.31 \pm 6.6) ; \quad r^{2}=0.9926$

The standard deviation associated with this protocol was $\pm 6.6 \mathrm{~K}$ for 995 compounds using the melting temperatures of a total of 381 compounds to generate the appropriate slopes and intercepts. Three of the series listed in Table 2 were evaluated on the basis of only three points and were not included in this study. The histogram shown in Figure 11 illustrates the error associated with using this protocol.

While it may be desirable to use experimental melting data in establishing a correlation within a series, it may also be possible to use existing parameters such as some of those listed in Tables $1-3$ to predict melting in related series where no experimental data are available. Ubbelo- 


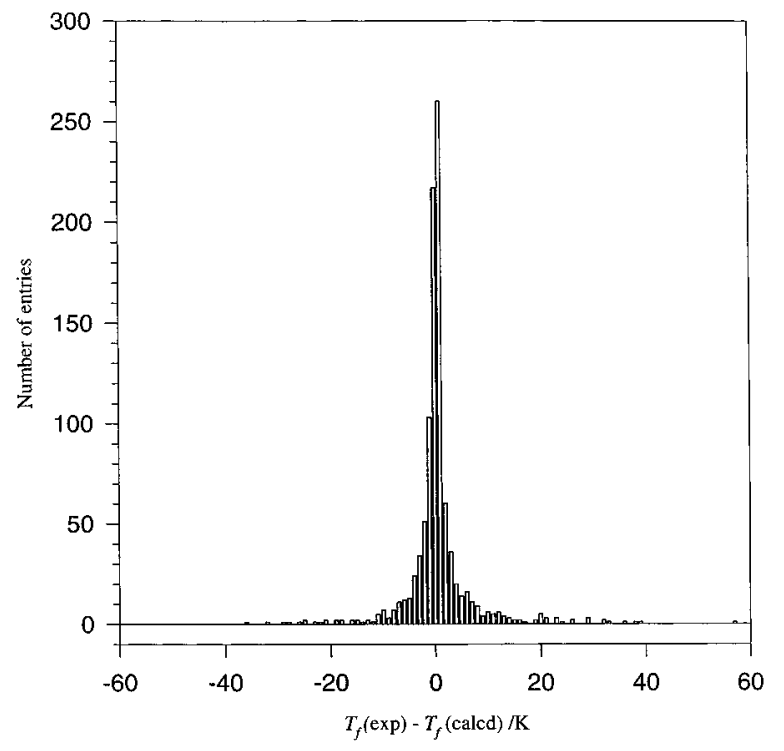

Figure 11. Distribution of errors based on the use of the melting temperature of the parent and three experimental data points to estimate the melting behavior of each series for 995 compounds.

hde ${ }^{21}$ has previously shown that, for a series of isomeric $\mathrm{n}$-heptadecanones and isomeric n-octadecanones, the melting temperatures of each respective isomer were usually within a few degrees of each other with the following exceptions. Symmetrical di-n-octyl ketone and the methyl ketones exhibited higher melting temperatures than their isomeric counterparts: in this case about $10 \mathrm{~K}$. Correlation equations derived to predict the melting behavior of odd and even 4-alkanones could be used to predict the melting of 4-n-heptadecanone and 4-n-octadecanone. These equations could also be used to evaluate the melting temperatures of all other odd and even unsymmetrical aliphatic ketones with more than nine carbon atoms in which the carbonyl group was located at an internal position in the molecule. While such a possibility needs further testing, if these observations are general, it should be possible to devel op appropriate correlation equations based on only a few data points that can be universally applied to predict the melting behavior of other related homologous series.

\section{Discussion}

The correlations observed in this study suggest that the melting temperatures of homologous series can be predicted. A few simple mathematical relationships serve to provide a quantitative measure of the melting behavior of molecules of any homologous series characterized by the same symmetry number. The results clearly demonstrate that melting temperature is not a group property. Furthermore, although the primary focus thus far has been on the modeling melting behavior, the results also provide some insight into some of the fundamental factors that govern this behavior. Reference has al ready been made to the importance of crystal packing. Additionally, the hyperbolic behavior observed can be understood from a phenomenological perspective by the following thermodynamic considerations.

The melting temperature is the point at which the increase in the population of states associated with the available degrees of freedom is sufficient to overcome the attractive forces between mol ecules in the crystal. The total phase change entropy associated in going from a rigid solid to an isotropic liquid upon melting (i.e. the sum of all the entropy changes associated with phase changes), $\Delta_{\text {tpce }} S$, has been shown to be a group property. ${ }^{22}$ As a group property, $\Delta_{\text {tpce }} \mathrm{S}$ can be expressed in terms of eq 9 , where $A_{s}$ is the

$$
\Delta_{\text {tpce }} \mathrm{S}\left(\mathrm{T}_{\mathrm{f}}\right)=\left(\mathrm{A}_{\mathrm{s}}\right) \mathrm{n}+\left(\mathrm{B}_{\mathrm{s}}\right) \mathrm{J} \cdot \mathrm{mol}^{-1} \cdot \mathrm{K}^{-1}
$$

contribution of the repeating group, $\mathrm{n}$ refers to the number of such groups, and B refers to the sum of the contributions of the remaining portions of the molecule. For the $n$-alkane and dialkylarsinic acid series, eq 9 can be rewritten in the form of eqs 10 and 11 , respectively.

$$
\begin{gathered}
\Delta_{\text {tpce }} \mathrm{S}\left(\mathrm{T}_{\mathrm{f}}\right)=(9.3) \mathrm{n}+(35.2) \mathrm{J} \cdot \mathrm{mol}^{-1} \cdot \mathrm{K}^{-1} \\
\Delta_{\text {tpce }} \mathrm{S}\left(\mathrm{T}_{\mathrm{f}}\right)=2(9.3) \mathrm{n}+(11.2) \mathrm{J} \cdot \mathrm{mol}^{-1} \cdot \mathrm{K}^{-1}
\end{gathered}
$$

The use of group values evaluated for the anal ogous total phase change enthalpy (i.e. the total enthalpy change associated in going from a rigid solid at $0 \mathrm{~K}$ to the isotropic liquid at the melting temperature, $\Delta_{\text {tpce }} \mathrm{H}$ ), is more problematic. Group values for total phase change enthal py are not al ways transferable from one series to another. Total phase change enthalpies can even decrease within a series. ${ }^{22}$ However, group values often do seem to work moderately well within some homologous series. A plot of experimental $\Delta_{\text {tpce }} \mathrm{H}$ versus the number of consecutive methylene groups, $n$, using the n-alkanes and di-n-alkylarsinic acids as examples, results in the following relationships:

$$
\begin{aligned}
\left.\Delta_{\text {tpce }} \mathrm{H}\left(\mathrm{T}_{\mathrm{f}}\right) /\right] \cdot \mathrm{mol}^{-1}= & \mathrm{n}(3725 \pm 38)-(1838 \pm 7500) ; \\
& (37 \text { data points }) \mathrm{r}^{2}=0.9964(12) \\
\left.\Delta_{\text {tpce }} \mathrm{H}\left(\mathrm{T}_{\mathrm{f}}\right) /\right] \cdot \mathrm{mol}^{-1}= & 2 \mathrm{n}(3348 \pm 66)-(9512 \pm 2800) ; \\
& (17 \text { data points }) \mathrm{r}^{2}=0.9941
\end{aligned}
$$

The fusion temperature can be evaluated from Gibbs equation as the ratio of the enthal py to the entropy change associated with melting. In this case, using total phase change properties works best: expressing phase change enthal py and entropy in terms of expressions that emphasize their group properties, eq 14, performing the necessary

$$
T_{f}=\Delta_{\text {tpce }} H / \Delta_{\text {tpce }} S=\left(A_{H} n+B_{H}\right) /\left(A_{S} n+B_{S}\right)
$$

division, and factoring the quotient results in eq 15 . The

$$
T_{f}=\frac{B_{H}}{B_{S}}+\left[\frac{A_{H}}{A_{S}}-\frac{B_{H}}{B_{S}}\right]\left[\sum_{x=0}^{\infty}\left(\frac{B_{S}}{A_{S} n}\right)^{2 x}-\sum_{x=0}^{\infty}\left(\frac{B_{S}}{A_{S} n}\right)^{2 x+1}\right]
$$

terms $A$ and $B$ refer to the slope and intercept of each respective empirical correlation, and the subscripts $\mathrm{H}$ and $\mathrm{S}$ serve to identify the enthalpic and entropic terms, respectively, while $\mathrm{n}$ refers to the number of consecutive repeat units in the series.

Equation 15 expresses the melting temperature in terms of a series of sums and differences of the ratios of slopes and intercepts of each respective group relationship. The hyperbolic dependence of temperature on the number of repeat groups, $n$, is easily demonstrated by this equation. As the number of repeat units increases, the bracketed terms on the right approach one and the melting temperature asymptotically approaches the ratio $A_{H} / A_{s}$. For the $n$-alkanes and di-n-alkylarsinic acids, the ratio according to the values of $A_{H}$ and $A_{S}$ in eqs $9-12$ is 405 and $360 \mathrm{~K}$, respectively. Depending on the relative magnitudes of the 


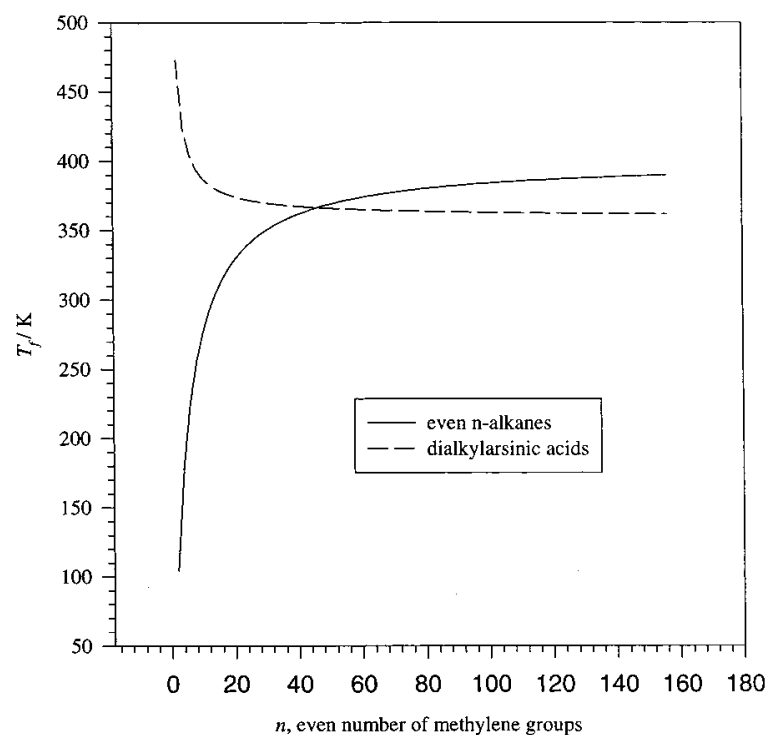

Figure 12. Melting point behavior of the even n-alkanes and the dialkylarsinic acids of formula $\left[\mathrm{CH}_{3}\left(\mathrm{CH}_{2}\right)_{n}\right]_{2} \mathrm{AsOH}$ when calculated as a ratio of the total phase change enthalpy to the total phase change entropy. Both properties were estimated by group additivity.

slopes and intercepts, eq 13 can behave either as an ascending or descending hyperbolic function. This is illustrated in Figure 12 using the ratio of eqs 12 to 10 and 13 to 11 .

\section{Summary}

The correlations observed in this study suggest that the melting of homologous series can be predicted successfully. The results observed clearly demonstrate that melting temperature is not a group property. Even materials exhibiting liquid crystal behavior appear to be modeled successfully, provided the appropriate transitions are correlated. While this work has focused on predicting melting as a function of size and less on uncovering the fundamental factors governing melting behavior, the results do provide some insight and guidance. The simplicity of the mathematics used to model this behavior is encouraging, suggesting similar features in the theoretical factors governing this property. All of the systems examined, including those forming liquid crystals, involve rod shaped molecules. The correlations observed appear to be a consequence of comparing series of related molecules likely to pack similarly in a crystal lattice. The anomalous behavior observed for the parent and the first few members of a series may al so be a consequence of this factor. Packing in these molecules is most likely to be influenced by the nature of the "functional group". As the number of repeat units increases, however, this influence is attenuated and in the limit approaches the melting behavior of the polymer the series resembles.

\section{Acknowledgment}

We would like to thank Professors Dave Larson and J oel Liebman for valuable comments and suggestions.

\section{Supporting Information Available:}

Tables including the experimental melting points, references, and melting points calculated using the protocols described in the text. This material is available free of charge via the Internet at http://pubs.acs.org.

\section{Literature Cited}

(1) Ubbelohde, A. Melting and Crystal Structure; Oxford University Press: London, 1965.

(2) Flory, P.J . Thermodynamics of Crystallization of High Polymers, IV. J. Chem. Phys. 1949, 17, 223.

(3) Flory, P. J .; Vrij, A. Melting points of linear chain homologues. The normal paraffin hydrocarbons. J . Am. Chem. Soc. 1963, 85, 3548.

(4) Wunderlich, B.; Czornyj, G. A study of equilibrium melting of polyethylene. Macromol ecules 1977, 10, 906-13.

(5) Buckley, C. P.; Kovacs, A. J. Melting behavior of low molecular weight poly(ethylene oxide fractions. I. Extended chain crystals. Prog. Colloid Polym. Sci. 1975, 58, 44.

(6) Mandelkern, L.; Stack, G. N. Equilibrium melting temperature of long chain molecules. Macromol ecules 1984, 1, 871 and references cited.

(7) Lutton, E. S. Thermal Properties and Crystal State. In Fatty Acids, Part 4, 2nd ed.; Markley, K. S., Ed.; Interscience Publishers: New York, 1967; Chapter XXII.

(8) Yalkowski, S. H.; Krzyzaniak, J. F.: Myrdal, P. B. Relationships between melting point and boiling point of organic compounds. Ind. Eng. Chem. 1994, 33, 1872.

(9) Tsakanikas, P. D.; Yalkowsky, S. H. Estimation of the melting point of flexible molecules: Aliphatic hydrocarbons. Toxicol. Environ. Chem. 1988, 17, 19.

(10) Krzyzaniak, J . F.; Myrdal, P. B.; Simamora, P.; Yalkowski, S. H. Boiling point and melting point prediction for aliphatic nonhydrogen bonding compounds. Ind. Eng. Chem. 1995, 34, 2530.

(11) Katritzky, A. R.; Lobanov, V. S.; Karelson, M. QSPR: The correlation and quantitative prediction of chemical and physical properties from structure. Chem. Soc. Rev. 1995, 279.

(12) Gavezzotti, A. Molecular symmetry. Melting temperatures and melting enthal pies of substituted benzenes and naphthalenes. J Chem. Soc., Perkin Trans. 2 1995, 1399.

(13) Shriner, R. L.; Herman, C. F. K.; Morrill, T. C.; Curtin, D. Y.; Fuson, R. C. TheSystematic I dentification of Organic Compounds, 7th ed.; Wiley: New York, 1998.

(14) Aldrich Catalog Handbook of Fine Chemicals; Aldrich Chemical Co.: Milwaukee, WI, 2000.

(15) The 2000 Catalog; Lancaster Synthesis Inc.: PO Box 1000 , Windham, NH 03087, 2000.

(16) TRC Thermodynamic Tables, Non-Hydrocarbons; Marsh, K. N. (Director); The Texas A\&M University System: College Station, TX, 1991; Supplement 64.

(17) Benson, S. W. Thermochemical Kinetics; Methods for the Estimation of Thermochemical Data and Rate Parameters, 2nd ed.; Wiley: New York, 1976.

(18) Zeberg-Mikkelsen, C. K.; Stenby, E. H. Predicting the melting points and enthalpies of fusion of saturated triglycerides by a group contribution method. Fluid Phase Equilib. 1999, 162, 7-17.

(19) Chickos, J. S. Heats of Sublimation. In Molecular Structure and Energetics; Liebman, J . F., Greenberg, A., Eds.; VCH Publishers: New York, 1987; Vol. 2, Chapter 3.

(20) Gray, G. W.; J ones, B. J.; Marson, F. Mesomorphism and Chemical Constitution. Part VII. The Effect of Halogen Substitution on the Mesomorphism of the trans-p-n-Alkoxycinnamic Acids. J. Chem. Soc. 1956, 1417.

(21) Barrall, E. M., II; J ohnson, J . F. Thermal Properties of Liquid Crystals. In Liquid Crystals and Plastic Crystals; Gray, G. W., Winsor, P. A., Eds.; Ellis Horwood Limited: Chichester, U.K., 1974; Vol. 2, Chapter 10.

(22) Chickos, J . S.; Acree, W. E., J r.; Liebman, J . F. Estimating SolidLiquid Phase Change Enthal pies and Entropies. J . Phys. Chem. Ref. Data 1999, 28, 1535-1674.

(23) Brandrup, J ., Immergut, E. H., Ed. Polymer Handbook, 3rd ed.; Wiley: New York, 1989

(24) Stephenson, R. M.; Malonowski, S. Handbook of the Thermodynamics of Organic Compounds; Elsevier: New York, 1987.

(25) Dreisbach, D. Physical Properties of Chemical Compounds, Vol. I-III; Advances in Chemistry Series 15, 22, 29; American Chemical Society: Washington, DC, 1955, 1959, 1961.

(26) Beilstein Handbook of Organic Chemistry; Springer-Verlag: New York.

(27) Lutton, E. S. In Fatty Acids, Their Chemistry, Properties, Production and Uses; Markley, K. S., Ed.; Wiley: New York, 1967; Part 4.

(28) Markley, K. S. In Fatty Acids, Their Chemistry, Properties, Production and Uses; Markley, K. S., Ed.; Wiley: New York, 1967; Part 4.

(29) Ralston, A. W. Fatty Acids and Their Derivatives; Wiley: New York, 1948

(30) Rappoport, Z., Ed. CRC Handbook of Tables for Organic Compound I dentification, 3rd ed.; CRC Press: Boca Raton, FL, 1967.

(31) The Merck Index, 7th ed.; Merck and Co.: Rahway, NJ , 1960.

(32) Carfagna, C.; Vacatello, M.; Corradini, P. Melting behavior of a series of monoamides. Thermochim. Acta 1979, 28, 265.

(33) Gray, G. W.; Hartley, J . B.; J ones, B. Mesomorphism and chemical constitution. Part V. The mesomorphic properties of the $4^{\prime}-n-$ 
alkoxydiphenyl-4-carboxylic Acids and their simple alkyl esters. . Chem. Soc. 1955, 1412

(34) Gray, G. W.; J ones, B. J .; Marson, F. Mesomorphism and Chemical Constitution. The Effect of Halogen Substitution on the Mesomorphism of the trans-p-n-Alkoxycinnamic Acids. J . Chem. Soc. 1956, 1417.

(35) Gondova, T.; Kralik, P.; Gonda, J. Determination of Some Thermodynamic Characteristics of Melting of 8-Alkyltheophyllines by the DSC Method. Thermochim. Acta 1989, 156, 147.

(36) Gray, G. W.; J ones, B. J . The Mesomorphic Transition Points of the p-n-Alkoxybenzoic Acids. A Correction. J . Chem. Soc. 1953, 4179.

(37) Gray, G. W. I ones, B. J. Mesomorphism and chemical constitution. The Effect of Halogen Substitution on the Mesomorphism of the p-n-Alkoxybenzoic Acids. J . Chem. Soc. 1954, 2556.
(38) Gray, G. W.; J ones, B. J . Mesomorphism and chemical constitution. Part I. The n-alkoxynaphthoic acids. J. Chem. Soc. 1954 683.

(39) Barrall, E. M., II; J ohnson, J . F. In Liquid Crystals and Plastic Crystals; Gray, G. W., Winsor, P. A., Eds.; Ellis Horwood Limited: Chichester, U.K., 1974; Vol. 2, Chapter 10.

(40) Starkweather, W., J r. Melting and crystalline transitions in normal perfluoroalkanes and poly(tetrafluoroethylene). Macromolecules 1986, 19, 1131

Received for review J uly 27, 2000. Accepted J anuary 4, 2001. We would like to thank the University of Missouri-St. Louis Graduate School for a summer fellowship to G.N.

J E0002235 